TITLE:

\title{
Poisson mechanics for perturbed MIC-Kepler problems at both positive and negative energies
}

$\operatorname{AUTHOR}(S):$

Iwai, Toshihiro; Matsumoto, Shogo

\section{CITATION:}

Iwai, Toshihiro ... [et al]. Poisson mechanics for perturbed MIC-Kepler problems at both positive and negative energies. Journal of Physics A: Mathematical and Theoretical 2012, 45(36): 365203.

ISSUE DATE:

2012-08-22

URL:

http://hdl.handle.net/2433/160218

\section{RIGHT:}

C 2012 IOP Publishing Ltd.; この論文は出版社版でありません。引用の 際には出版社版をご確認ご利用ください。; This is not the published version. Please cite only the published version. 


\title{
Poisson mechanics for perturbed MIC-Kepler problems at both positive and negative energies
}

\author{
Toshihiro Iwai and Shogo Matsumoto \\ Department of Applied Mathematics and Physics, \\ Kyoto University, Kyoto, Japan
}

\begin{abstract}
The MIC-Kepler problem, an extension of the Kepler problem, is known to admit the symmetry group $S U(2) \times S U(2)$ or $S L(2, \mathbb{C})$, according as the energy is negative or positive. In general, each of co-adjoint orbits of a Lie group carries the canonical symplectic form called the KKS form, and a Hamiltonian dynamical system is defined on it if a suitable Hamiltonian is given. Perturbed MIC-Kepler problems can be treated in this setting if a perturbed Hamiltonian in normal form is determined according to whether the energy is negative or positive. Since the co-adjoint orbit in question can be viewed as a symplectic leaf of the associated Lie algebra $\mathfrak{s u}(2) \oplus \mathfrak{s u}(2)$ or $\mathfrak{s l}(2, \mathbb{C})$ according as the energy is negative or positive, the perturbed MIC-Kepler problems in normal form can be described in the Poisson mechanics defined on respective symmetry Lie algebras. Thus, the equations of motion for perturbed systems can be described in the form of Poisson brackets for both cases of $\mathfrak{s u}(2) \oplus \mathfrak{s u}(2)$ and $\mathfrak{s l}(2, \mathbb{C})$ on an equal footing. It will be shown further how two parameters assigning a co-adjoint orbit of $S U(2) \times S U(2)$ or $S L(2, \mathbb{C})$ are related to the parameters contained in the MIC-Kepler problem. The perturbation of the MIC-Kepler problem to be treated in this article is rather restricted to that caused by the presence of weak constant electric and magnetic fields orthogonal to each other. When regularized, the perturbed Hamiltonians at both positive and negative energies are put in Birkhoff-Gustavson normal form and thereby the flows generated by the perturbed Hamiltonians are studied in Poisson mechanics in terms of variables associated with constants of motion for the MIC-Kepler problem.
\end{abstract}




\section{Introduction}

As is well known, according to a book of Guillemin and Sternberg [1], the "completed" space of orbits for the Kepler problem of negative energy is topologically $S^{2} \times S^{2}$. Then, an integrable approximation to a perturbed Kepler problem of negative energy should be defined to be a dynamical system on $S^{2} \times S^{2}$. Since $S^{2} \times S^{2}$ is a co-adjoint orbit of the symmetry group $S O(4)$ (or its covering group $S U(2) \times S U(2))$ for the Kepler problem of negative energy, it admits a canonical symplectic structure (the KKS form [2]), which is shown to be a restriction of the Poisson structure on $\mathfrak{s o}(3) \oplus \mathfrak{s o}(3) \cong \mathfrak{s o}(4)$. Within this framework, a number of studies have been done for perturbed Kepler problems of negative energy with emphasis on monodromy associated with an energy-momentum mapping (see [3] and references therein). The perturbation of the hydrogen atom (or the Kepler problem) caused by small static electric and magnetic fields orthogonal to each other is studied in [4-6]. The perturbations caused by small constant electric and magnetic fields which are non-orthogonal to each other are also studied in [7-11].

The idea on treatment of perturbed Kepler problems in a similar way to that mentioned above can be applied for perturbed Kepler problems of positive energy as well. As is expected, any integrable approximation to a perturbed Kepler problem of positive energy should be described on a co-adjoint orbit of the symmetry group $S L(2, \mathbb{C})$, a covering group of $S O_{0}(3,1)$. In [12], one of the authors studied the symmetry of the MIC-Kepler problem, an extension of the Kepler problem, from the viewpoint of a dynamical group $U(2,2)$ and showed that its symmetry group is realized as a subgroup isomorphic to $S U(2) \times S U(2), S L(2, \mathbb{C})$, or $S U(2) \ltimes \mathbb{R}^{3}$, according as the energy is negative, positive, or zero, where MIC is an abbreviation of McIntosh-Cisneros [13]. In addition, the above-mentioned idea on treatment of perturbed MIC-Kepler problems was stated in Sec. 7 of [12] but not performed then.

From the viewpoint of the symmetry group associated with an unperturbed dynamical system, the MIC-Kepler problem is preferable to the Kepler problem. This is because the co-adjoint orbits of the symmetry group $S U(2) \times S U(2)$ or $S L(2, \mathbb{C})$ are assigned by two parameters, with which the two parameters of the MIC-Kepler problem are related, but the Kepler problem has one parameter only, where the two parameters of the MIC-Kepler problem are the strength of the attractive potential and the strength of the monopole field (or the strength of the repulsive potential) and the parameter of the Kepler problem is of course the strength of the attractive potential. The monopole might sound unrealistic 
in the ordinary sense, but the monopole field is rather natural. In fact, in the regularization of the Kepler problem, one uses the so-called KS-transformation, which is viewed as a realization of the projection map of the $S O(2)$ bundle $\dot{\mathbb{R}}^{4} \rightarrow$ $\dot{\mathbb{R}}^{3}$, where $\dot{\mathbb{R}}^{4}=\mathbb{R}^{4} \backslash\{0\}$ and $\dot{\mathbb{R}}^{3}=\mathbb{R}^{4} \backslash\{0\}$. Further, the $S O(2)$ bundle $\dot{\mathbb{R}}^{4} \rightarrow \dot{\mathbb{R}}^{3}$ has a natural connection, whose curvature coincides with the monopole field. A similar situation occurs for a planar three-body system. In fact, the cotangent bundle $T^{*} \dot{\mathbb{R}}^{4}$ for a planar three-body center-of-mass system is reduced to the phase space diffeomorphic with $T^{*} \dot{\mathbb{R}}^{3}$, on which the reduced symplectic form is the standard one on $T^{*} \dot{\mathbb{R}}^{3}$ plus a monopole filed, where the monopole field serves as the curvature form of the canonical connection mentioned above [14]. In addition, the monopole field with a quantized strength physically comes out if a center-ofmass system for three particles on a plane is reduced by using the $S O(2)$ symmetry to set up a line bundle on which a Hamiltonian operator is defined [15].

This article, a successor to [12], shows that when regularized, perturbed MICKepler problems can be put in Birkhoff-Gustavson (BG) normal form [16-19] in the Poisson mechanics associated with the symmetry Lie algebra $\mathfrak{s u}(2) \oplus \mathfrak{s u}(2)$ or $\mathfrak{s l}(2, \mathbb{C})$ according as the energy is negative or positive. By the use of a dynamical group $U(2,2)$ which includes $S U(2) \times S U(2)$ and $S L(2, \mathbb{C})$ as subgroups, perturbed MIC-Kepler problems of both negative and positive energies can be treated, on an equal footing, in Poisson mechanics on $\mathfrak{s u}(2) \oplus \mathfrak{s u}(2)$ and on $\mathfrak{s l}(2, \mathbb{C})$, respectively. In fact, $\mathfrak{s u}(2) \oplus \mathfrak{s u}(2)$ and $\mathfrak{s l}(2, \mathbb{C})$ are realized as Lie subalgebras of $\mathfrak{u}(2,2)$, and further the vector space $\mathbb{R}^{6}$ can be endowed with an exterior product operation, $\wedge^{-}$or $\wedge^{+}$, so as to be isomorphic with $\mathfrak{s u}(2) \oplus \mathfrak{s u}(2)$ or $\mathfrak{s l}(2, \mathbb{C})$ as Lie algebras; $(\mathfrak{s u}(2) \oplus \mathfrak{s u}(2),[\cdot, \cdot]) \cong\left(\mathbb{R}^{6}, \wedge^{-}\right)$or $(\mathfrak{s l}(2, \mathbb{C}),[\cdot, \cdot]) \cong\left(\mathbb{R}^{6}, \wedge^{+}\right)$, and then Poisson brackets are defined accordingly in each case. The co-adjoint orbits of the symmetry groups $S U(2) \times S U(2)$ and $S L(2, \mathbb{C})$ are realized as symplectic leaves of respective Lie algebras. From the viewpoint of the normal-form perturbation theory, the perturbation of the MIC-Kepler problem of zero energy is viewed as degenerate and is not treated in this article.

A model perturbed Hamiltonian in this article comes from the MIC-Kepler problem in the presence of weak constant electric and magnetic fields orthogonal to each other, after [3]. From the viewpoint of constructing perturbed Hamiltonian systems, a rather simple but relevant perturbation is preferable. For this reason, the perturbation caused by small non-orthogonal electric and magnetic fields is not considered in this article. Since perturbed Hamiltonian systems are treated on the co-adjoint orbits of the symmetry groups and since those orbits are assigned by two-parameters, difference between the MIC-Kepler and the Kepler problems will 
appear when they are perturbed. For example, the leading term of the perturbed MIC-Kepler Hamiltonian in BG normal form is shown to be an extension of that for the perturbed Kepler problem [3]. It will be shown that the Hamiltonian flow generated by the leading term for the perturbed Kepler problem is periodic, but that for the perturbed MIC-Kepler problem is not periodic in general.

The organization of this article is as follows: Sections 2 to 4 are reviews from [12]. Section 2 is a setting up for the MIC-Kepler problem which is defined as a reduced Hamiltonian system by a $U(1)$ action. Section 3 is concerned with the group $U(2,2)$ and its subgroups $S U(2) \times S U(2)$ and $S L(2, \mathbb{C})$, which are (covering groups of) the symmetry groups for the MIC-Kepler problem of negative and positive energies, respectively. Interrelations among components of the momentum map associated with $U(2,2)$ are given explicitly, which will be used in Sec. 5 in describing Poisson mechanics on the Lie algebras $\mathfrak{s u}(2) \oplus \mathfrak{s u}(2)$ and $\mathfrak{s l}(2, \mathbb{C})$. Section 4 deals with the reduction of the phase space $T^{*} \mathbb{R}^{4} \cong \mathbb{C}^{4}$ by the groups $U(1) \times U(1)$ and $U(1) \times \mathbb{R}$, where the first factor $U(1)$ of these product groups is associated with the so-called KS-transformation and the latter factors $U(1)$ and $\mathbb{R}$ denote the one-parameter groups generated by the Hamiltonian flows for the harmonic oscillator and for the repulsive oscillator, respectively. These Hamiltonian flows can be assigned to those for the MIC-Kepler problem at negative and positive energies, respectively. Section 5 contains dynamics on the co-adjoint orbits of $S U(2) \times S U(2)$ and of $S L(2, \mathbb{C})$. Hamiltonian systems on the respective co-adjoint orbits can be treated in Poisson mechanics on the Lie algebras $\mathfrak{s u}(2) \oplus \mathfrak{s u}(2)$ and $\mathfrak{s l}(2, \mathbb{C})$, respectively. It is of great use to make $\mathbb{R}^{6}$ into Lie algebras endowed with exterior products $\wedge^{\mp}$ so as to be isomorphic with respective Lie algebras; $(\mathfrak{s u}(2) \oplus \mathfrak{s u}(2),[\cdot, \cdot]) \cong\left(\mathbb{R}^{6}, \wedge^{-}\right)$and $(\mathfrak{s l}(2, \mathbb{C}),[\cdot, \cdot]) \cong\left(\mathbb{R}^{6}, \wedge^{+}\right)$, where the superscripts \pm are associated with the sign of the energy of the MIC-Kepler problem. Then, dynamics on the co-adjoint orbits can be described in terms of vectors in $\mathbb{R}^{6}$ in Poisson mechanics formalism with Casimir functions. In Section 6 , interest centers on the perturbation of the MIC-Kepler problem with emphasis on the expansion of perturbed Hamiltonians in BG normal form. The perturbation is caused by the presence of a weak constant electric and magnetic fields orthogonal to each other. In Section 7, the leading terms of the perturbation are mainly treated in the equations of motion for the perturbed systems. When the flows generated by the leading terms in the cases of negative and positive energies are periodic, the averaging of higher-order terms along the flow is touched upon as well. Section 8 is for concluding remarks together with a list of frequently used symbols. 


\section{Setting up}

We make a brief review of the MIC-Kepler problem after [12] with emphasis on iso-energetic orbit spaces.

\section{1 $S O(2)$ reduction}

This subsection shows that the so-called Kustaanheimo-Stiefel (KS) transformation is a realization of a reduction procedure with $S O(2)$.

Let $\dot{\mathbb{R}}^{4}:=\mathbb{R}^{4} \backslash\{0\}$ and let $T^{*} \dot{\mathbb{R}}^{4} \cong \dot{\mathbb{R}}^{4} \times \mathbb{R}^{4}$ be the phase space endowed with the Cartesian coordinates $\left(x_{j}, y_{j}\right), j=1, \cdots, 4$. The standard symplectic form on $T^{*} \dot{\mathbb{R}}^{4}$ is given by

$$
d \theta \quad \text { with } \quad \theta=\sum_{j=1}^{4} y_{j} d x_{j} .
$$

The $S O(2)$ acts on $\left(T^{*} \dot{\mathbb{R}}^{4}, d \theta\right)$ symplectically in the manner

$$
(x, y) \mapsto(T(t) x, T(t) y), \quad t \in \mathbb{R}
$$

where

$$
T(t)=\left(\begin{array}{cc}
R(t) & 0 \\
0 & R(t)
\end{array}\right) \quad \text { with } \quad R(t)=\left(\begin{array}{cc}
\cos t & -\sin t \\
\sin t & \cos t
\end{array}\right) .
$$

Let $\mathfrak{s o}(2)$ denote the Lie algebra of $S O(2)$. The momentum map $\Phi: T^{*} \dot{\mathbb{R}}^{4} \rightarrow$ $\mathfrak{s o}(2)^{*}$ associated with the $S O(2)$ action is defined through

$$
\theta\left(\xi_{M}(x, y)\right)=\langle\Phi(x, y), \xi\rangle
$$

where $\langle\cdot, \cdot\rangle$ denote the paring, and where $\xi_{M}$ is the infinitesimal generator defined on $M=T^{*} \dot{\mathbb{R}}^{4}$ for $\xi \in \mathfrak{s o}(2)$ by

$$
\xi_{M}(x, y)=\left.\frac{d}{d t}(T(t) x, T(t) y)\right|_{t=0} .
$$

Since $\mathfrak{s o}^{*}(2) \cong \mathbb{R}$, the $\Phi(x, y)$ is viewed as a real-valued function and proves to take the form

$$
\Phi(x, y)=-x_{2} y_{1}+x_{1} y_{2}-x_{4} y_{3}+x_{3} y_{4} .
$$

Since the $S O(2)$ action is free and proper, the quotient space $\Phi^{-1}(\mu) / S O(2)$ becomes a manifold. Let

$$
\pi_{\mu}: \Phi^{-1}(\mu) \rightarrow \Phi^{-1}(\mu) / S O(2), \quad \iota_{\mu}: \Phi^{-1}(\mu) \rightarrow T^{*} \dot{\mathbb{R}}^{4}
$$


be the natural projection and the inclusion map, respectively. The $\left(T^{*} \dot{\mathbb{R}}^{4}, d \theta\right)$ reduces to the symplcetic manifold $\left(\Phi^{-1}(\mu) / S O(2), \sigma_{\mu}\right)$, where $\sigma_{\mu}$ is determined through

$$
\pi_{\mu}^{*} \sigma_{\mu}=\iota_{\mu}^{*} d \theta
$$

The reduced phase space $\Phi^{-1}(\mu) / S O(2)$ is shown to be diffeomorphic to $T^{*} \dot{\mathbb{R}}^{3}$, which is realized through the Kustaanheimo-Stiefel (KS) transformation,

$$
M_{K S}=\left(\begin{array}{cccc}
x_{3} & x_{4} & x_{1} & x_{2} \\
-x_{4} & x_{3} & x_{2} & -x_{1} \\
x_{1} & x_{2} & -x_{3} & -x_{4} \\
-x_{2} & x_{1} & -x_{4} & x_{3}
\end{array}\right)
$$

along with the relations

$$
\left(\begin{array}{l}
r_{1} \\
r_{2} \\
r_{3} \\
r_{4}
\end{array}\right)=M_{K S}\left(\begin{array}{l}
x_{1} \\
x_{2} \\
x_{3} \\
x_{4}
\end{array}\right), \quad\left(\begin{array}{l}
p_{1} \\
p_{2} \\
p_{3} \\
p_{4}
\end{array}\right)=\frac{1}{2 r} M_{K S}\left(\begin{array}{l}
y_{1} \\
y_{2} \\
y_{3} \\
y_{4}
\end{array}\right)
$$

where $\left(r_{k}, p_{k}\right) \in \dot{\mathbb{R}}^{3} \times \mathbb{R}^{3}$ with $k=1,2,3$ and

$$
r_{4}=0, \quad p_{4}=\frac{\Phi}{2 \rho}, \quad \rho=\sum_{j=1}^{4} x_{j}^{2}=\sqrt{\sum_{k=1}^{3} r_{k}^{2}} .
$$

In terms of $\left(r_{k}, p_{k}\right)$, the reduced symplectic form $\sigma_{\mu}$ is put in the form

$$
\sigma_{\mu}=\sum_{k=1}^{3} d p_{k} \wedge d r_{k}-\frac{\mu}{2 r^{3}}\left(r_{1} d r_{2} \wedge d r_{3}+r_{2} d r_{3} \wedge d r_{1}+r_{3} d r_{1} \wedge d r_{2}\right)
$$

As was mentioned in Introduction, the second term of the right-hand side of the above equation is $\mu$ times the curvature of the canonical connection for the $S O(2)$ bundle $\dot{\mathbb{R}}^{4} \rightarrow \dot{\mathbb{R}}^{3}$.

\subsection{The MIC-Kepler problem}

This subsection starts with the definition of the MIC-Kepler problem as a reduced Hamiltonian system and then provides the iso-energetic orbit spaces for the MICKepler problem. 
The conformal Kepler problem is a dynamical system $\left(T^{*} \dot{\mathbb{R}}^{4}, d \theta, H_{c}\right)$, where $H_{c}$ is the Hamiltonian defined by

$$
H_{c}=\frac{1}{2}\left(\frac{1}{4 \rho} \sum_{j=1}^{4} y_{j}^{2}\right)-\frac{\kappa}{\rho}, \quad \rho=\sum_{j=1}^{4} x_{j}^{2},
$$

and where $\kappa>0$ is a constant. Since this Hamiltonian system is $S O(2)$ invariant, it is reduced to a Hamiltonian system $\left(T^{*} \dot{\mathbb{R}}^{3}, \sigma_{\mu}, H_{\mu}\right)$, which is called the MICKepler problem [20], where $H_{\mu}$ is defined through $\pi_{\mu}^{*} H_{\mu}=\iota_{\mu}^{*} H_{c}$ and expressed in terms of $\left(r_{k}, p_{k}\right)$ as

$$
H_{\mu}=\frac{1}{2} \sum_{k=1}^{3} p_{k}^{2}+\frac{\mu^{2}}{8 \rho^{2}}-\frac{\kappa}{\rho} .
$$

We note here that the parameter $\mu$ (or $\mu^{2} / 8$ ) serves as the strength of the repulsive potential, which is a natural consequence of the fact that $\mu$ is the conserved angular momentum associated with the $S O(2)$ symmetry.

The conformal Kepler problem is closely related with the harmonic oscillator, the repulsive oscillator, and a free particle. To see this, we introduce the Hamiltonians defined on $\mathbb{R}^{4} \times \mathbb{R}^{4}$ by

$$
\begin{aligned}
A_{\lambda} & =\frac{1}{2} \sum_{j=1}^{4} y_{j}^{2}+\frac{\lambda^{2}}{2} \sum_{j=1}^{4} x_{j}^{2}, \\
R_{\lambda} & =\frac{1}{2} \sum_{j=1}^{4} y_{j}^{2}-\frac{\lambda^{2}}{2} \sum_{j=1}^{4} x_{j}^{2}, \\
F & =\frac{1}{2} \sum_{j=1}^{4} y_{j}^{2},
\end{aligned}
$$

where $\lambda$ is a positive parameter. Hamiltonians $A_{\lambda}, R_{\lambda}, F$ are related to $H_{c}$ by

$$
\begin{aligned}
4 \rho\left(H_{c}+\frac{\lambda^{2}}{8}\right) & =A_{\lambda}-4 \kappa, \\
4 \rho\left(H_{c}-\frac{\lambda^{2}}{8}\right) & =R_{\lambda}-4 \kappa, \\
4 \rho H_{c} & =F-4 \kappa .
\end{aligned}
$$

From the above equations, we have three types of energy manifolds for $H_{c}$,

$$
H_{c}^{-1}(E)=\left\{\begin{array}{lll}
A_{\lambda}^{-1}(4 \kappa) & \text { if } & E=-\lambda^{2} / 8<0, \\
R_{\lambda}^{-1}(4 \kappa) & \text { if } & E=\lambda^{2} / 8>0, \\
F^{-1}(4 \kappa) & \text { if } & E=0 .
\end{array}\right.
$$


Strictly speaking, the left-hand side is not exactly equal to the right-hand side in the above equation, because $H_{c}^{-1}(E)$ is a submanifold of $T^{*} \dot{\mathbb{R}}^{4}$ and $A_{\lambda}^{-1}(4 \kappa)$, $R_{\lambda}^{-1}(4 \kappa), F^{-1}(4 \kappa)$ are those of $T^{*} \mathbb{R}^{4}$. However, $H_{c}^{-1}(E)$ can be completed so that the above equation may hold for a fixed value of $E<0, E>0$, or $E=0$. In other words, we can take into account a singular point $r=0$ as long as the total energy $E$ is conserved at $r=0$ as well.

The Hamiltonian flow of $H_{c}$ on $H_{c}^{-1}(E)$ corresponds to the Hamiltonian flow of $A_{\lambda}, R_{\lambda}$, or $F$, depending on whether the energy is negative, positive or zero;

$$
4 \rho X_{H_{c}}= \begin{cases}X_{A_{\lambda}}, & \text { if } \quad E=-\lambda^{2} / 8<0, \\ X_{R_{\lambda}}, & \text { if } \quad E=\lambda^{2} / 8>0 \\ X_{F}, & \text { if } \quad E=0 .\end{cases}
$$

The respective energy manifolds for the conformal Kepler problem project to those for the MIC-Kepler manifold through the projection $\pi_{\mu}$;

$$
H_{\mu}^{-1}(E)=\left\{\begin{array}{lll}
\left(A_{\lambda}^{-1}(4 \kappa) \cap \Phi^{-1}(\mu)\right) / S O(2) & \text { if } \quad E=-\lambda^{2} / 8<0, \\
\left(R_{\lambda}^{-1}(4 \kappa) \cap \Phi^{-1}(\mu)\right) / S O(2) & \text { if } \quad E=\lambda^{2} / 8>0, \\
\left(F^{-1}(4 \kappa) \cap \Phi^{-1}(\mu)\right) / S O(2) & \text { if } \quad E=0 .
\end{array}\right.
$$

From (15) it follows that the Hamiltonian flow $\exp \left(t X_{H_{c}}\right)$ of the conformal Kepler problem can be viewed, after changing of the time parameter, as the Hamiltonian flow $\exp \left(t X_{A_{\lambda}}\right), \exp \left(t X_{R_{\lambda}}\right)$, or $\exp \left(t X_{F}\right)$, according to whether the energy is negative, $E=-\lambda^{2} / 8$, positive, $E=\lambda^{2} / 8$, or zero $E=0$. Since the Hamiltonian flow $\exp \left(t X_{H_{c}}\right)$ projects to that, $\exp \left(t H_{\mu}\right)$, of the MIC-Kepler problem, and since the $S O(2)$ action commutes with the flow $\exp \left(t X_{H_{c}}\right)$, the Hamiltonian flow $\exp \left(t X_{H_{\mu}}\right)$ on the energy manifold $H_{\mu}^{-1}(E)$ can be looked on as that projected from $\exp \left(t X_{A_{\lambda}}\right), \exp \left(t X_{R_{\lambda}}\right)$, or $\exp \left(t X_{F}\right)$, according to whether the energy is negative, $E=-\lambda^{2} / 8$, positive, $E=\lambda^{2} / 8$, or zero $E=0$. We denote the one-parameter groups expressed as $\exp \left(t X_{A_{\lambda}}\right), \exp \left(t X_{R_{\lambda}}\right)$, and $\exp \left(t X_{F}\right)$, by $S O(2), \mathbb{R}$, and $\mathbb{R}$, respectively. Consequently, we obtain the following proposition.

Proposition 2.1 The iso-energetic orbit space for the MIC-Kepler problem is given by

$$
\begin{aligned}
& H_{\mu}^{-1}(E) /\left\{\exp \left(t X_{H_{\mu}}\right)\right\} \\
= & \left\{\begin{array}{lll}
\left(A_{\lambda}^{-1}(4 \kappa) \cap \Phi^{-1}(\mu)\right) /(S O(2) \times S O(2)) & \text { if } \quad E=-\lambda^{2} / 8<0, \\
\left(R_{\lambda}^{-1}(4 \kappa) \cap \Phi^{-1}(\mu)\right) /(S O(2) \times \mathbb{R}) & \text { if } \quad E=\lambda^{2} / 8>0, \\
\left(F^{-1}(4 \kappa) \cap \Phi^{-1}(\mu)\right) /(S O(2) \times \mathbb{R}) & \text { if } \quad E=0 .
\end{array}\right.
\end{aligned}
$$


Since the topology of $H_{\mu}^{-1}(E) /\left\{\exp \left(t X_{H_{\mu}}\right)\right\}$ depends only on the sign of $E$, we are allowed to put $\lambda=1$ in studying the symmetry of the MIC-Kepler problem.

\section{$3 \quad U(2,2)$ as a dynamical group}

As $S U(2,2)$ or $S O(4,2)$ is known as a dynamical group for the MIC-Kepler problem, we study $U(2,2)$, taking into account the $S O(2)$ action (2) associated with the KS transformation, after [12].

\subsection{The group $U(2,2)$ and its momentum map}

This subsection deals with the momentum map associated with $U(2,2)$ together with its components expressed explicitly.

We introduce a complex vector space structure on $\mathbb{R}^{4} \times \mathbb{R}^{4}$ through the bijection $\phi: \mathbb{R}^{4} \times \mathbb{R}^{4} \rightarrow \mathbb{C}^{4}$ defined by

$$
w=\left(\begin{array}{l}
w_{1} \\
w_{2} \\
w_{3} \\
w_{4}
\end{array}\right)=C_{1}\left(\begin{array}{l}
x_{1} \\
x_{2} \\
x_{3} \\
x_{4}
\end{array}\right)+C_{2}\left(\begin{array}{l}
y_{1} \\
y_{2} \\
y_{3} \\
y_{4}
\end{array}\right)
$$

where

$$
C_{1}=\frac{1}{2}\left(\begin{array}{cccc}
1 & i & 1 & i \\
-1 & -i & 1 & i \\
1 & i & 1 & i \\
-1 & -i & 1 & i
\end{array}\right), \quad C_{2}=\frac{1}{2}\left(\begin{array}{cccc}
-i & 1 & -i & 1 \\
i & -1 & -i & 1 \\
i & -1 & i & -1 \\
-i & 1 & i & -1
\end{array}\right)
$$

Let $D:=\left(D_{j k}\right)=\operatorname{diag}(1,1,-1,-1)$. We introduce on $\mathbb{C}^{4}$ the 1 -form

$$
\Theta:=\frac{i}{2} \operatorname{tr}\left(D w d w^{*}\right)=\frac{i}{2} \sum_{j, k=1}^{4} D_{j k} w_{k} d \bar{w}_{j} .
$$

We note here that since $w$ is a column vector the product, $w d w^{*}$, of $w$ and the Hermitian conjugate $d w^{*}$ of $d w$ forms a matrix. From the definition of $\Theta$, we easily verify that

$$
d \theta=d \Theta
$$

where the $\theta$ is the standard one-form given in (1). With this in mind, in what follows we take $\left(\mathbb{C}^{4}, d \Theta\right)$ as our symplectic space in place of $\left(T^{*} \mathbb{R}^{4}, d \theta\right)$. 
The $S O(2)$ action given in (2) can be rewritten as a diagonal action of $U(1)$ on $\mathbb{C}^{4}$

$$
\operatorname{diag}\left(e^{i t}, e^{i t}, e^{i t}, e^{i t}\right),
$$

and the momentum map $\Phi$ given in (3) as

$$
\Phi(w)=\frac{i}{2} \operatorname{tr}\left(D w(i w)^{*}\right)=\frac{1}{2}\left(\left|w_{1}\right|^{2}+\left|w_{2}\right|^{2}-\left|w_{3}\right|^{2}-\left|w_{4}\right|^{2}\right) .
$$

In what follows, we refer to the $S O(2)$ action (2) as the $U(1)$ action.

Now we consider the group

$$
U(2,2)=\left\{g \in M_{4}(\mathbb{C}) \mid g^{*} D g=D\right\},
$$

where $M_{4}(\mathbb{C})$ denotes the space of $4 \times 4$ complex matrices. The $U(2,2)$ action on $\mathbb{C}^{4}$ is symplectic and preserves the momentum map $\Phi$, as is easily verified from Eqs.(20) and (23). Note that the $U(1)$ given in (22) is a subgroup of $U(2,2)$.

Let $\mathfrak{u}(2,2)$ denote the Lie algebra of $U(2,2)$, which is endowed with the inner product defined as

$$
\gamma(\xi, \eta)=\frac{1}{2} \operatorname{tr}\left(\xi^{*} \eta\right), \quad i \xi, i \eta \in \mathfrak{u}(2,2) .
$$

Through this inner product, the dual space $\mathfrak{u}(2,2)^{*}$ is identified with $\mathfrak{u}(2,2)$. We can define the momentum map associated with the $U(2,2)$ action,

$$
i J: \mathbb{C}^{4} \rightarrow \mathfrak{u}(2,2)^{*} \cong \mathfrak{u}(2,2),
$$

through

$$
\Theta\left(i \xi_{P}(w)\right)=\frac{1}{2} \operatorname{tr}\left(\xi^{*} D w w^{*}\right)=\gamma(\xi, J(w)),
$$

where $i \xi \in \mathfrak{u}(2,2), P=\mathbb{C}^{4}$, and where $i \xi_{P}$ is the infinitesimal generator defined by

$$
i \xi_{P}(w)=\left.\frac{d}{d t} \exp (i t \xi) \cdot w\right|_{t=0}, \quad w \in \mathbb{C}^{4}
$$

Through (26), the momentum map $i J$ is expressed as

$$
J(w)=D w w^{*} .
$$


We take a basis, $\left\{i e_{\ell}\right\}, \ell=0,1, \cdots, 15$, of $\mathfrak{u}(2,2)$ as follows:

$$
\begin{array}{rlr}
e_{0} & =\frac{1}{2}\left(\begin{array}{cc}
\sigma_{0} & 0 \\
0 & \sigma_{0}
\end{array}\right), & e_{1}=\frac{1}{2}\left(\begin{array}{cc}
\sigma_{0} & 0 \\
0 & -\sigma_{0}
\end{array}\right), \\
e_{j+1} & =\left(\begin{array}{cc}
\sigma_{j} & 0 \\
0 & 0
\end{array}\right), & e_{j+4}=\left(\begin{array}{cc}
0 & 0 \\
0 & \sigma_{j}
\end{array}\right), \\
e_{8} & =\frac{1}{2}\left(\begin{array}{cc}
0 & \sigma_{0} \\
-\sigma_{0} & 0
\end{array}\right), & e_{j+8}=\frac{1}{2}\left(\begin{array}{cc}
0 & \sigma_{j} \\
-\sigma_{j} & 0
\end{array}\right), \\
e_{12} & =\frac{i}{2}\left(\begin{array}{cc}
0 & \sigma_{0} \\
\sigma_{0} & 0
\end{array}\right), & e_{j+12}=\frac{i}{2}\left(\begin{array}{cc}
0 & \sigma_{j} \\
\sigma_{j} & 0
\end{array}\right),
\end{array}
$$

where $\sigma_{0}$ is the $2 \times 2$ identity matrix and $\sigma_{j}, j=1,2,3$, are the Pauli spin matrices. Note that $\mathfrak{u}(2,2) \cong \mathfrak{u}(1) \oplus \mathfrak{s u}(2,2)$ and that $e_{0}$ is a base of $\mathfrak{u}(1)$ and that $e_{1}, \cdots, e_{15}$ form a basis of $\mathfrak{s u}(2,2)$. The components $J_{\ell}=\gamma\left(e_{\ell}, J\right), \ell=0,1, \cdots, 15$, of the momentum map $i J$ are expressed as

$$
\begin{aligned}
J_{0}(w) & =\frac{1}{4}(\langle u, u\rangle-\langle v, v\rangle), & J_{1}(w) & =\frac{1}{4}(\langle u, u\rangle+\langle v, v\rangle), \\
J_{j+1}(w) & =\frac{1}{2}\left\langle u, \sigma_{j} u\right\rangle, & J_{j+4}(w) & =-\frac{1}{2}\left\langle v, \sigma_{j} v\right\rangle, \\
J_{8}(w) & =\frac{1}{2} \operatorname{Re}\langle v, u\rangle, & J_{j+8}(w) & =\frac{1}{2} \operatorname{Re}\left\langle v, \sigma_{j} u\right\rangle \\
J_{12}(w) & =\frac{1}{2} \operatorname{Im}\langle v, u\rangle, & J_{j+12}(w) & =\frac{1}{2} \operatorname{Im}\left\langle v, \sigma_{j} u\right\rangle,
\end{aligned}
$$

where

$$
u=\left(\begin{array}{l}
w_{1} \\
w_{2}
\end{array}\right), \quad v=\left(\begin{array}{c}
w_{3} \\
w_{4}
\end{array}\right), \quad j=1,2,3,
$$

and where $\langle$,$\rangle denotes the Hermitian inner product on \mathbb{C}^{2}$.

\subsection{Symmetry groups for the MIC-Kepler problem}

In this subsection, symmetry groups for the MIC-Kepler problem are shown to be realized as subgroups of $U(2,2)$. The associated symmetry algebras are also given, to which constants of motions are assigned together with relations among them.

As is already mentioned in Introduction, the symmetry group for the zeroenergy is not reviewed here. We start by noting that among $J_{\ell}$ there are functions of special interest. The first one is

$$
2 J_{0}=\Phi .
$$


Further, we find that the Hamiltonians for the harmonic oscillator and for the repulsive oscillator are members of $J_{\ell}$;

$$
\begin{aligned}
2 J_{1}(w) & =\frac{1}{2} \sum_{j=1}^{4} y_{j}^{2}+\frac{1}{2} \sum_{j=1}^{4} x_{j}^{2}=A_{1}, \\
-2 J_{8}(w) & =\frac{1}{2} \sum_{j=1}^{4} y_{j}^{2}-\frac{1}{2} \sum_{j=1}^{4} x_{j}^{2}=R_{1},
\end{aligned}
$$

where $A_{1}$ and $R_{1}$ are the Hamiltonians for the harmonic oscillator and the repulsive oscillator, respectively (see (12) with $\lambda=1$ ). The Hamiltonian flows associated with Hamiltonians (31) determine one-parameter groups acting on $\mathbb{C}^{4}$,

$$
\begin{aligned}
e^{2 i t e_{1}} & =\left(\begin{array}{cc}
e^{i t} \sigma_{0} & 0 \\
0 & e^{-i t} \sigma_{0}
\end{array}\right), \\
e^{-2 i t e_{8}} & =\left(\begin{array}{cc}
\cosh t \sigma_{0} & -i \sinh t \sigma_{0} \\
i \sinh t \sigma_{0} & \cosh t \sigma_{0}
\end{array}\right),
\end{aligned}
$$

respectively, where $e_{1}$ and $e_{8}$ are the $4 \times 4$ matrices given in (27). These groups are isomorphic to $U(1)$ and $\mathbb{R}$, respectively, and commute with the $U(1)$ action given in $(2)$ or $(22)$, since $\left[e_{0}, e_{1}\right]=\left[e_{0}, e_{8}\right]=0$.

The symmetry groups for the Hamiltonians (31), which are subgroups of $S U(2,2)$ that commute with $(32)$, turn out to be, respectively,

$$
\begin{aligned}
& G^{-}:=\left\{\left(\begin{array}{ll}
A & 0 \\
0 & B
\end{array}\right) \mid A, B \in S U(2)\right\} \cong S U(2) \times S U(2), \\
& G^{+}:=\left\{\left(\begin{array}{cc}
A & B \\
-B & A
\end{array}\right) \mid \begin{array}{l}
A^{*} A-B^{*} B=\sigma_{0}, A^{*} B+B^{*} A=0, \\
\operatorname{det}(A+i B)=1
\end{array}\right\} \cong S L(2, \mathbb{C}) .
\end{aligned}
$$

Since the groups $G^{-}$and $G^{+}$commute with the $U(1)$ given by (22), their actions project to those on the respective reduced energy manifolds, $\left(A_{1}^{-1}(4 \kappa) \cap\right.$ $\left.\Phi^{-1}(\mu)\right) / U(1)$ and $\left(R_{1}^{-1}(4 \kappa) \cap \Phi^{-1}(\mu)\right) / U(1)$. From (16) with $S O(2) \cong U(1)$, it then turns out that $G^{-}$and $G^{+}$are symmetry groups for the MIC-Kepler problem of energy $E<0$ and $E>0$, respectively.

Let $\mathcal{G}^{-}$and $\mathcal{G}^{+}$be the Lie algebras of $G^{-}$and $G^{+}$, respectively, which are 
expressed as

$$
\begin{aligned}
& \mathcal{G}^{-}=\left\{i \xi=\left(\begin{array}{cc}
i \xi_{1} & 0 \\
0 & i \xi_{2}
\end{array}\right) \mid i \xi_{k} \in \mathfrak{s u}(2), k=1,2\right\} \cong \mathfrak{s u}(2) \oplus \mathfrak{s u}(2), \\
& \mathcal{G}^{+}=\left\{i \xi=\left(\begin{array}{cc}
i \xi_{1} & i \xi_{2} \\
-i \xi_{2} & i \xi_{1}
\end{array}\right) \mid i \xi_{k} \in \mathfrak{s u}(2), k=1,2\right\} \cong \mathfrak{s l}(2, \mathbb{C}),
\end{aligned}
$$

respectively.

The Lie algebra $\mathcal{G}^{-}$has a basis $\left\{i e_{j+1}, i e_{j+4}\right\}, j=1,2,3$, to which the functions $J_{j+1}, J_{j+4}, j=1,2,3$, are associated. We note that they are related by

$$
\begin{aligned}
& \left(J_{0}+J_{1}\right)^{2}=J_{2}^{2}+J_{3}^{2}+J_{4}^{2}, \\
& \left(J_{0}-J_{1}\right)^{2}=J_{5}^{2}+J_{6}^{2}+J_{7}^{2},
\end{aligned}
$$

and that the left-hand sides of the above equations are constant on the intersection $A_{1}^{-1}(4 \kappa) \cap \Phi^{-1}(\mu)$.

The Lie algebra $\mathcal{G}^{+}$has a basis $\left\{i e_{j+1}+i e_{j+4}, i e_{j+8}\right\}, j=1,2,3$, to which the functions $J_{j+1}+J_{j+4}, J_{j+8}, j=1,2,3$, are associated together with the relations

$$
\begin{aligned}
4\left(J_{0}^{2}-J_{8}^{2}\right) & =\left(J_{2}+J_{5}\right)^{2}+\left(J_{3}+J_{6}\right)^{2}+\left(J_{4}+J_{7}\right)^{2}-4\left(J_{9}^{2}+J_{10}^{2}+J_{11}^{2}\right), \\
2 J_{0} J_{8} & =\left(J_{2}+J_{5}\right) J_{9}+\left(J_{3}+J_{6}\right) J_{10}+\left(J_{4}+J_{7}\right) J_{11}
\end{aligned}
$$

where the left-hand sides of the above equations are constant on the intersection $R_{1}^{-1}(4 \kappa) \cap \Phi^{-1}(\mu)$.

In a summary, we have the following proposition.

Proposition 3.1 Among subgroups of $U(2,2)$, the groups $U(1) \times U(1)$ and $S U(2) \times$ $S U(2)$, which commute to each other, are associated with the MIC-Kepler problem of negative energy. The group $U(1) \times U(1)$ has the corresponding momentum map given by $\Phi \oplus A_{1}$ or $2 J_{0} \oplus 2 J_{1}$, and the group $S U(2) \times S U(2)$ serves as the symmetry group, whose momentum map has the components $J_{j+1}, J_{j+4}, j=1,2,3$, together with two mutual relations given in (35). With the MIC-Kepler problem of positive energy, associated are the groups $U(1) \times \mathbb{R}$ and $S L(2, \mathbb{C})$, which commute to each other. The group $U(1) \times \mathbb{R}$ has the corresponding momentum map given by $\Phi \oplus R_{1}$ or $-2 J_{0} \oplus 2 J_{8}$, and the group $S L(2, \mathbb{C})$, serving as the symmetry group, has the associated momentum map which has the components $J_{j+1}+J_{j+4}, J_{j+8}, j=1,2,3$, together with two mutual relations given in (36). Here the expressions of $J_{k}$ are given in (28). 


\section{Reduction by $U(1) \times U(1)$ and $U(1) \times \mathbb{R}$}

In this section, after [12] we treat the reduction of $\mathbb{C}^{4}$ by two product groups; (i) $U(1) \times U(1)$, the product of $(22)$ and $(32 \mathrm{a})$, (ii) $U(1) \times \mathbb{R}$, the product of $(22)$ and (32b). The associated momentum maps are expressed as (i) $\Phi \oplus A_{1}$ and (ii) $\Phi \oplus R_{1}$, respectively (see Prop. 3.1). Since the symmetry groups $S U(2) \times S U(2)$ and $S L(2, \mathbb{C})$ commute with $U(1) \times U(1)$ and $U(1) \times \mathbb{R}$, respectively, these symmetry groups acts on the respective reduced phase space.

\subsection{Iso-energetic orbit space $M_{\mu, \kappa}^{-}$at a negative energy}

This subsection shows that the iso-energetic orbit space $H_{\mu}^{-1}(E) /\left\{\exp t X_{H_{\mu}}\right\}$ for the MIC-Kepler problem of negative energy is realized as a co-adjoint orbit of the symmetry group $S U(2) \times S U(2)$. According to Prop. 2.1, we start by reducing the phase space $\mathbb{C}^{4}$ by the action of the group $U(1) \times U(1)$. We denote the reduced phase space $\left(A_{1}^{-1}(4 \kappa) \cap \Phi^{-1}(\mu)\right) /(U(1) \times U(1))$ by $M_{\mu, \kappa}^{-}$, and call it an iso-energetic orbit space.

Let $\iota_{\mu, \kappa}^{-}$and $\pi_{\mu, \kappa}^{-}$be the inclusion map and the natural projection, respectively;

$$
\begin{aligned}
\iota_{\mu, \kappa}^{-}: A_{1}^{-1}(4 \kappa) \cap \Phi^{-1}(\mu) & \rightarrow \mathbb{C}^{4}, \\
\pi_{\mu, \kappa}^{-}: A_{1}^{-1}(4 \kappa) \cap \Phi^{-1}(\mu) & \rightarrow M_{\mu, \kappa}^{-}:=\left(A_{1}^{-1}(4 \kappa) \cap \Phi^{-1}(\mu)\right) /(U(1) \times U(1)) .
\end{aligned}
$$

The symplectic form $\sigma_{\mu, \kappa}^{-}$on $M_{\mu, \kappa}^{-}$is defined through

$$
\left(\pi_{\mu, \kappa}^{-}\right)^{*} \sigma_{\mu, \kappa}^{-}=\left(\iota_{\mu, \kappa}^{-}\right)^{*} d \theta
$$

In the below, we will realize $M_{\mu, \kappa}^{-}$as a (co-)adjoint orbit of the symmetry group $G^{-} \cong S U(2) \times S U(2)$. First, we note that if restricted to $\mathcal{G}^{-} \cong \mathfrak{s u}(2) \oplus \mathfrak{s u}(2)$, the inner product $(25)$ for $i \xi=i \xi_{1} \oplus i \xi_{2}, i \eta=i \eta_{1} \oplus i \eta_{2} \in \mathcal{G}^{-}$is put in the form

$$
\gamma^{-}(\xi, \eta)=\frac{1}{2} \operatorname{tr}\left(\xi_{1}^{*} \eta_{1}\right)+\frac{1}{2} \operatorname{tr}\left(\xi_{2}^{*} \eta_{2}\right)
$$

which induces the inner product on $\mathcal{G}^{-}$, and thereby $\mathcal{G}^{-}$and its dual are identified. The function (26) for $i \xi=i \xi_{1} \oplus i \xi_{2}$ turns into

$$
\frac{1}{2} \operatorname{tr}\left(\xi^{*} D w w^{*}\right)=\frac{1}{2} \operatorname{tr}\left(u u^{*} \xi_{1}-v v^{*} \xi_{2}\right) .
$$

From (39) and (40), the momentum map associated with $G^{-} \cong S U(2) \times S U(2)$,

$$
i K^{-}=i K_{L}^{-} \oplus i K_{R}^{-}: \mathbb{C}^{4} \cong \mathbb{C}^{2} \times \mathbb{C}^{2} \rightarrow \mathcal{G}^{-} \cong \mathfrak{s u}(2) \oplus \mathfrak{s u}(2),
$$


is expressed as

$$
K^{-}(w)=\left(\begin{array}{cc}
u u^{*} & 0 \\
0 & -v v^{*}
\end{array}\right)-\frac{1}{2}\left(\begin{array}{cc}
\langle u, u\rangle \sigma_{0} & 0 \\
0 & -\langle v, v\rangle \sigma_{0}
\end{array}\right)
$$

together with

$$
K_{L}^{-}(u)=u u^{*}-\frac{1}{2}\langle u, u\rangle \sigma_{0}, \quad K_{R}^{-}(v)=-v v^{*}+\frac{1}{2}\langle v, v\rangle \sigma_{0},
$$

where $u$ and $v$ are column vectors given in (29) and then the symbols $u u^{*}$ and $v v^{*}$ denote $2 \times 2$ complex matrices.

As is easily verified, $K^{-}=K_{L}^{-} \oplus K_{R}^{-}$is Ad-equivariant with respect to $G^{-}$and invariant with respect to $U(1) \times U(1)$;

$$
\begin{aligned}
K^{-}(g w) & =\operatorname{Ad}_{g} K^{-}(w), \quad g \in G^{-}, \\
K^{-}\left(e^{i t} w\right) & =K^{-}(w), \quad K^{-}\left(e^{2 i t e_{1}} w\right)=K^{-}(w), \quad t \in \mathbb{R} .
\end{aligned}
$$

For $u_{0}=(\sqrt{4 \kappa+\mu}, 0)^{T}$ and $v_{0}=(\sqrt{4 \kappa-\mu}, 0)^{T}$, we can easily show that

$$
A_{1}^{-1}(4 \kappa) \cap \Phi^{-1}(\mu)=\left\{\left(g u_{0}, h v_{0}\right) \mid(g, h) \in S U(2) \times S U(2)\right\} .
$$

It then follows that

$$
\begin{aligned}
& K^{-}\left(A_{1}^{-1}(4 \kappa) \cap \Phi^{-1}(\mu)\right) \\
= & \left\{\operatorname{Ad}_{g} K_{L}^{-}\left(u_{0}\right) \oplus \operatorname{Ad}_{h} K_{R}^{-}\left(v_{0}\right) \mid(g, h) \in S U(2) \times S U(2)\right\} .
\end{aligned}
$$

This implies that the image of $A_{1}^{-1}(4 \kappa) \cap \Phi^{-1}(\mu)$ by $K^{-}$is an adjoint orbit in $\mathfrak{s u}(2) \oplus \mathfrak{s u}(2)$, which we denote by $\mathcal{O}^{-}$.

Any co-adjoint orbit is endowed with a symplectic form, called the KirillovKostant-Souriau (KKS) form. The KKS form on the (co-)adjoint orbit $\mathcal{O}^{-}$is defined at $\nu \in \mathcal{O}^{-}$to be

$$
\begin{aligned}
\omega^{-}\left(\xi_{Q}, \eta_{Q}\right)(\nu) & =-\frac{i}{2} \operatorname{tr}\left(\nu^{*}[\xi, \eta]\right) \\
& =-\frac{i}{2} \operatorname{tr}\left(\nu_{1}^{*}\left[\xi_{1}, \eta_{1}\right]\right)-\frac{i}{2} \operatorname{tr}\left(\nu_{2}^{*}\left[\xi_{2}, \eta_{2}\right]\right),
\end{aligned}
$$

where $\xi=\xi_{1} \oplus \xi_{2}, \eta=\eta_{1} \oplus \eta_{2}, \nu=\nu_{1} \oplus \nu_{2}$ with $\xi_{1}, \cdots, \nu_{2}$ being $2 \times 2$ traceless Hermitian matrices, and where $i \xi_{Q}$ and $i \eta_{Q}$ are the infinitesimal generators of the (co-)adjoint action on $Q:=\mathcal{G}^{-}$defined by

$$
i \xi_{Q}(i \nu)=\left.\frac{d}{d t} \operatorname{Ad}_{\exp (i t \xi)}(i \nu)\right|_{t=0}, \quad i \eta_{Q}(i \nu)=\left.\frac{d}{d t} \operatorname{Ad}_{\exp (i t \eta)}(i \nu)\right|_{t=0},
$$


respectively.

Since $K^{-}$is $U(1) \times U(1)$ invariant, it projects to the map

$$
\tilde{K}_{\mu, \kappa}^{-}: M_{\mu, \kappa}^{-}=\left(A_{1}^{-1}(4 \kappa) \cap \Phi^{-1}(\mu)\right) /(U(1) \times U(1)) \rightarrow \mathcal{O}^{-}
$$

through

$$
\tilde{K}_{\mu, \kappa}^{-} \circ \pi_{\mu, \kappa}^{-}(w)=K^{-} \circ \iota_{\mu, \kappa}^{-}(w), \quad w \in A_{1}^{-1}(4 \kappa) \cap \Phi^{-1}(\mu) .
$$

We can prove that $\tilde{K}_{\mu, \kappa}^{-}$is a symplectomorphism, that is, $\tilde{K}_{\mu, \kappa}^{-}$is a diffeomorphism and symplectic; $\left(\tilde{K}_{\mu, \kappa}^{-}\right)^{*} \omega^{-}=\sigma_{\mu, \kappa}^{-}$. We come to the following proposition.

Proposition 4.1 The iso-energetic orbit space $\left(M_{\mu, \kappa}^{-}, \sigma_{\mu, \kappa}^{-}\right)$for the MIC-Kepler problem of negative energy (see $(17)$ with $E<0$ ) can be realized as a (co-)adjoint orbit, $\left(\mathcal{O}^{-}, \omega^{-}\right)$, of the symmetry group $G^{-} \cong S U(2) \times S U(2)$ together with the KKS form $\omega^{-}$. The isomorphism is shown in the following commutative diagram:

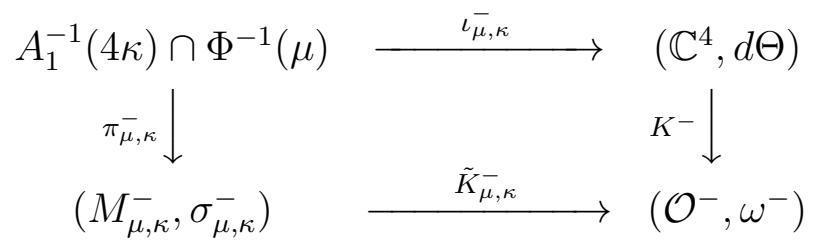

\subsection{Iso-energetic orbit space $M_{\mu, \kappa}^{+}$at a positive energy}

In this subsection, the iso-energetic orbit space $H_{\mu}^{-1}(E) /\left\{\exp t X_{H_{\mu}}\right\}$ for the MICKepler problem at a positive energy is realized as a co-adjoint orbit of the symmetry group $S L(2, \mathbb{C})$. Our task is to reduce the phase space $\mathbb{C}^{4}$ by using $U(1) \times \mathbb{R}$, the product of (22) and (32b). The resultant phase space $\left(R_{1}^{-1}(4 \kappa) \cap \Phi^{-1}(\mu)\right) /(U(1) \times$ $\mathbb{R})$ is called an iso-energetic orbit space and denoted by $M_{\mu, \kappa}^{+}$.

Let $\iota_{\mu, \kappa}^{+}$and $\pi_{\mu, \kappa}^{+}$be the inclusion map and the natural projection, respectively;

$$
\begin{aligned}
\iota_{\mu, \kappa}^{+}: R_{1}^{-1}(4 \kappa) \cap \Phi^{-1}(\mu) \rightarrow \mathbb{C}^{4}, \\
\pi_{\mu, \kappa}^{+}: R_{1}^{-1}(4 \kappa) \cap \Phi^{-1}(\mu) \rightarrow M_{\mu, \kappa}^{+}:=\left(R_{1}^{-1}(4 \kappa) \cap \Phi^{-1}(\mu)\right) /(U(1) \times \mathbb{R}) .
\end{aligned}
$$

The symplectic form $\sigma_{\mu, \kappa}^{+}$on $M_{\mu, \kappa}^{+}$is defined through

$$
\left(\pi_{\mu, \kappa}^{+}\right)^{*} \sigma_{\mu, \kappa}^{+}=\left(\iota_{\mu, \kappa}^{+}\right)^{*} d \theta
$$

For $i \xi, i \eta \in \mathcal{G}^{+}$, the inner product $(25)$ is restricted to induce

$$
\gamma^{+}(\xi, \eta)=\operatorname{tr}\left(\xi_{1}^{*} \eta_{1}+\xi_{2}^{*} \eta_{2}\right)
$$


which equips $\mathcal{G}^{+}$with an inner product, and thereby $\mathcal{G}^{+}$and its dual are identified. Further, for $i \xi \in \mathcal{G}^{+}$, the function (26) turns into

$$
\frac{1}{2} \operatorname{tr}\left(\xi^{*} D w w^{*}\right)=\frac{1}{2} \operatorname{tr}\left(\left(u u^{*}-v v^{*}\right) \xi_{1}+\left(u v^{*}+v u^{*}\right) \xi_{2}\right) .
$$

From (53) and (54), the momentum map associated with $G^{+} \cong S L(2, \mathbb{C})$,

$$
i K^{+}: \mathbb{C}^{4} \rightarrow \mathcal{G}^{+} \cong \mathfrak{s l}(2, \mathbb{C})
$$

is expressed as

$$
K^{+}(w)=\frac{1}{2}\left(\begin{array}{cc}
U & V \\
-V & U
\end{array}\right)-\frac{1}{4}\left(\begin{array}{cc}
(\operatorname{tr} U) \sigma_{0} & (\operatorname{tr} V) \sigma_{0} \\
-(\operatorname{tr} V) \sigma_{0} & (\operatorname{tr} U) \sigma_{0}
\end{array}\right)
$$

where

$$
U=u u^{*}-v v^{*}, V=u v^{*}+v u^{*} .
$$

As is verified straightforward, $K^{+}$is Ad-equivariant with respect to $G^{+}$and invariant with respect to $U(1) \times \mathbb{R}$;

$$
\begin{aligned}
K^{+}(g w) & =\operatorname{Ad}_{g^{-1 *}} K^{+}(w), \quad g \in G^{+}, \\
K^{+}\left(e^{i t} w\right) & =K^{+}(w), \quad K^{+}\left(e^{-2 i t e s} w\right)=K^{+}(w), \quad t \in \mathbb{R} .
\end{aligned}
$$

Let $w_{0}$ be a fixed point of $R_{1}^{-1}(4 \kappa) \cap \Phi^{-1}(\mu)$ given by

$$
w_{0}=\left(\begin{array}{l}
u_{0} \\
v_{0}
\end{array}\right) \quad \text { with } \quad u_{0}=\sqrt{2}\left(\begin{array}{c}
\sqrt{\mu}+i \sqrt{2 \kappa} \\
0
\end{array}\right), \quad v_{0}=\sqrt{2}\left(\begin{array}{c}
-i \sqrt{2 \kappa} \\
0
\end{array}\right) .
$$

Then, we can easily show that

$$
R_{1}^{-1}(4 \kappa) \cap \Phi^{-1}(\mu)=\left\{e^{i s} g w_{0} \mid g \in G^{+}, s \in \mathbb{R}\right\} .
$$

From (57b) and (58), it follows that

$$
K^{+}\left(R_{1}^{-1}(4 \kappa) \cap \Phi^{-1}(\mu)\right)=\left\{\operatorname{Ad}_{g^{-1 *}} K^{+}\left(w_{0}\right) \mid g \in G^{+}\right\} .
$$

This implies that the image of $R_{1}^{-1}(4 \kappa) \cap \Phi^{-1}(\mu)$ by $K^{+}$is a (co-)adjoint orbit of $G^{+}$, which we denote by $\mathcal{O}^{+}$.

The KKS form on the (co-)adjoint orbit $\mathcal{O}^{+}$is defined at $\nu \in \mathcal{O}^{+}$to be

$$
\begin{aligned}
\omega^{+}\left(\xi_{Q}, \eta_{Q}\right)(\nu) & =-\frac{i}{2} \operatorname{tr}\left(\nu^{*}[\xi, \eta]\right) \\
& =-i \operatorname{tr}\left(\nu_{1}^{*}\left(\left[\xi_{1}, \eta_{1}\right]-\left[\xi_{2}, \eta_{2}\right]\right)+\nu_{2}^{*}\left(\left[\xi_{1}, \eta_{2}\right]+\left[\xi_{2}, \eta_{1}\right]\right)\right),
\end{aligned}
$$


where $i \nu, i \xi$, $i \eta \in \mathcal{G}^{+}$, and where $i \xi_{Q}$ and $i \eta_{Q}$ are the infinitesimal generators on $Q:=\mathcal{G}^{+}$defined by

$$
i \xi_{Q}(i \nu)=\left.\frac{d}{d t} \operatorname{Ad}_{\exp \left(-i t \xi^{*}\right)}(i \nu)\right|_{t=0}, \quad i \eta_{Q}(i \nu)=\left.\frac{d}{d t} \operatorname{Ad}_{\exp \left(-i t \eta^{*}\right)}(i \nu)\right|_{t=0},
$$

respectively.

Since $K^{+}$is $U(1) \times \mathbb{R}$ invariant, it projects to the map

$$
\tilde{K}_{\mu, \kappa}^{+}: M_{\mu, \kappa}^{+}=\left(R_{1}^{-1}(4 \kappa) \cap \Phi^{-1}(\mu)\right) /(U(1) \times \mathbb{R}) \rightarrow \mathcal{O}^{+}
$$

through

$$
\tilde{K}_{\mu, \kappa}^{+} \circ \pi_{\mu, \kappa}^{+}(w)=K^{+} \circ \iota_{\mu, \kappa}^{+}(w), \quad w \in R_{1}^{-1}(4 \kappa) \cap \Phi^{-1}(\mu) .
$$

We can prove that $\tilde{K}_{\mu, \kappa}^{+}$is a symplectomorphism, that is, $\tilde{K}_{\mu, \kappa}^{+}$is a diffeomorphism and $\left(\tilde{K}_{\mu, \kappa}^{+}\right)^{*} \omega^{+}=\sigma_{\mu, \kappa}^{+}$holds. The above discussion is summarized as follows:

Proposition 4.2 The iso-energetic orbit space $\left(M_{\mu, \kappa}^{+}, \sigma_{\mu, \kappa}^{+}\right)$for the MIC-Kepler problem of negative energy (see (17) with $E>0$ ) can be realized as a (co-)adjoint orbit, $\left(\mathcal{O}^{+}, \omega^{+}\right)$, of the symmetry group $G^{+} \cong S L(2, \mathbb{C})$ together with the KKS form $\omega^{+}$. The isomorphism is shown in the following diagram;

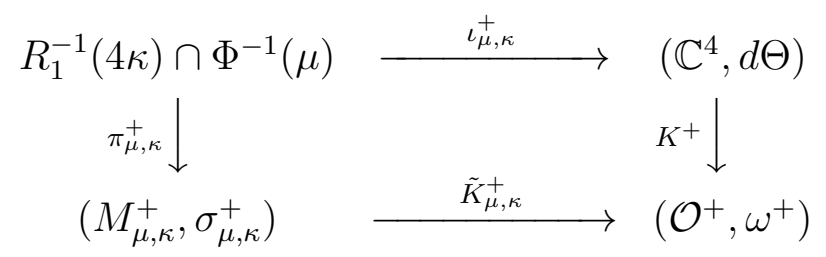

\section{Dynamics on co-adjoint orbits in $\mathcal{G}^{-}$and $\mathcal{G}^{+}$}

Though no dynamics remains to exist on the iso-energetic orbit space $M_{\mu, \kappa}^{-}$or $M_{\mu, \kappa}^{+}$for the MIC-Kepler problem, depending on $E<0$ or $E>0$, each isoenergetic orbit space can carry dynamics as a symplectic manifold endowed with the symplectic form $\omega_{\mu, \kappa}^{-}$or $\omega_{\mu, \kappa}^{+}$, if a suitable Hamiltonian is given on the isoenergetic orbit space in question. This happens if the MIC-Kepler problem is perturbed in such a way that the perturbed Hamiltonian is still invariant under the symmetry group, $U(1) \times U(1)$ or $U(1) \times \mathbb{R}$, associated with the reduction to each of the iso-energetic orbit spaces.

We discuss dynamics on the iso-energetic orbit spaces $M_{\mu, \kappa}^{-}$and $M_{\mu, \kappa}^{+}$in Poisson mechanics by introducing Poisson structures on $\mathcal{G}^{-}$and $\mathcal{G}^{+}$. 


\subsection{Co-adjoint orbits in $\mathcal{G}^{\mp}$}

In Secs.4.1 and 4.2, we have shown that the iso-energetic orbit spaces $\left(M_{\mu, \kappa}^{\mp}, \sigma_{\mu, \kappa}^{\mp}\right)$ are symplectomorphic to the (co-)adjoint orbits $\left(\mathcal{O}^{\mp}, \omega^{\mp}\right)$, respectively. However, to be precise, each of $\mathcal{O}^{\mp}$ is not a subset of $\mathcal{G}^{\mp}$ but of $i \mathcal{G}^{\mp}$. In this subsection, we treat (co-)adjoint orbits as subsets of $\mathcal{G}^{\mp}$.

Let

$$
i \mathcal{O}^{-}:=i K^{-}\left(\mathcal{A}_{1}^{-1}(4 \kappa) \cap \Phi^{-1}(\mu)\right), \quad i \mathcal{O}^{+}:=i K^{+}\left(\mathcal{R}_{1}^{-1}(4 \kappa) \cap \Phi^{-1}(\mu)\right) .
$$

Since $i \mathcal{O}^{-}$and $i \mathcal{O}^{+}$can be put in the form

$$
i \mathcal{O}^{-}=\left\{\operatorname{Ad}_{g} i K^{-}\left(w_{0}\right) \mid g \in G^{-}\right\}, \quad i \mathcal{O}^{+}=\left\{\operatorname{Ad}_{g^{-1 *}} i K^{+}\left(w_{0}\right) \mid g \in G^{+}\right\},
$$

respectively, $i \mathcal{O}^{\mp}$ are (co-)adjoint orbits of $G^{\mp}$ and are submanifolds of $\mathcal{G}^{\mp}$, respectively. Clearly, the $i \mathcal{O}^{\mp}$ are diffeomorphic to $\mathcal{O}^{\mp}$, respectively.

The KKS-forms $\Omega^{\mp}$ on $i \mathcal{O}^{\mp}$ at $i \nu \in i \mathcal{O}^{\mp}$ are defined to be

$$
\Omega^{\mp}\left(i \xi_{Q}(i \nu), i \eta_{Q}(i \nu)\right):=-\gamma^{\mp}(i \nu,[i \xi, i \eta]), \quad i \xi, i \eta \in \mathcal{G}^{\mp}
$$

respectively, where $i \xi_{Q}(i \nu), i \eta_{Q}(i \nu)$ are infinitesimal generators defined by (47) or (61), and where $\gamma^{\mp}$ are defined in (39) and (53), respectively.

It is shown in [12] that

$$
d \Theta\left(\xi_{P}(w), \eta_{P}(w)\right)=\omega^{\mp}\left(\xi_{Q}(\nu), \eta_{Q}(\nu)\right), \quad i \xi, i \eta \in \mathcal{G}^{\mp}, w \in \mathbb{C}^{4}
$$

where $\nu=K^{\mp}(w)$. If $i \xi_{P}(w)$ and $i \eta_{P}(w)$ are taken in place of $\xi_{P}(w)$ and $\eta_{P}(w)$, respectively, Eq.(66) along with the definition of $\omega^{\mp}$ and $\Omega^{\mp}$ is brought into

$$
d \Theta\left(i \xi_{P}(w), i \eta_{P}(w)\right)=\Omega^{\mp}\left(i \xi_{Q}(i \nu), i \eta_{Q}(i \nu)\right) .
$$

Since $i K_{*}^{\mp}\left(i \xi_{P}(w)\right)=i \xi_{Q}(i \nu)$, we can prove that $\left(M_{\mu, \kappa}^{\mp}, \sigma_{\mu, \kappa}^{\mp}\right)$ is symplectomorphic to $\left(i \mathcal{O}^{\mp}, \Omega^{\mp}\right)$ in the same manner as in [12].

\subsection{Poisson Structures on $\mathbb{R}^{6}$}

In this subsection, we define two Poisson manifolds $\left(\mathbb{R}^{6},\{,\}^{-}\right)$and $\left(\mathbb{R}^{6},\{,\}^{+}\right)$in association with $\mathcal{G}^{-}$and $\mathcal{G}^{+}$, respectively. For each of Poisson structures $\{,\}^{\mp}$, there exist two Casimir functions.

To begin with, we introduce Lie algebra structures on $\mathbb{R}^{6}$ in such a way that $\left(\mathbb{R}^{6}, \wedge^{\mp}\right)$ are isomorphic with $\mathcal{G}^{\mp}$ as Lie algebras, respectively, where $\wedge^{\mp}$ denote 
product operations to be defined soon. Let $\left\{b_{j}^{-}\right\}_{j=1, \cdots, 6}$ and $\left\{b_{j}^{+}\right\}_{j=1, \cdots, 6}$ denote the bases of $\mathcal{G}^{-}$and $\mathcal{G}^{+}$, respectively, defined by

$$
\begin{aligned}
b_{j}^{-} & =i e_{j+1}, \quad b_{j+3}^{-}=i e_{j+4}, \quad j=1,2,3, \\
b_{j}^{+} & =\frac{1}{2}\left(i e_{j+1}+i e_{j+4}\right), \quad b_{j+3}^{+}=i e_{j+8}, \quad j=1,2,3,
\end{aligned}
$$

where $\left\{i e_{\ell}\right\}, \ell=0,1, \cdots, 15$, is the basis of $\mathfrak{u}(2,2)$ given in $(27)$. The commutation relations among $b_{j}^{-}$and among $b_{j}^{+}$are given by

$$
\left\{\begin{array}{l}
{\left[b_{i}^{-}, b_{j}^{-}\right]=-2 \varepsilon_{i j k} b_{k}^{-},} \\
{\left[b_{i+3}^{-}, b_{j+3}^{-}\right]=-2 \varepsilon_{i j k} b_{k+3}^{-}, \quad i, j, k=1,2,3} \\
{\left[b_{i}^{-}, b_{j+3}^{-}\right]=0}
\end{array}\right.
$$

and

$$
\left\{\begin{array}{l}
{\left[b_{i}^{+}, b_{j}^{+}\right]=-\varepsilon_{i j k} b_{k}^{+},} \\
{\left[b_{i+3}^{+}, b_{j+3}^{+}\right]=\varepsilon_{i j k} b_{k}^{+}, \quad i, j, k=1,2,3} \\
{\left[b_{i}^{+}, b_{j+3}^{+}\right]=-\varepsilon_{i j k} b_{k+3}^{+}}
\end{array}\right.
$$

respectively. For the bases $\left\{b_{j}^{-}\right\}_{j=1, \cdots, 6}$ and $\left\{b_{j}^{+}\right\}_{j=1, \cdots, 6}$, the inner products among them are expressed as

$$
\begin{aligned}
& \gamma\left(b_{j}^{-}, b_{k}^{-}\right)=\delta_{j k}, \quad j, k=1,2, \cdots, 6, \\
& \gamma\left(b_{j}^{+}, b_{k}^{+}\right)=\frac{1}{2} \delta_{j k}, \quad j, k=1,2, \cdots, 6,
\end{aligned}
$$

respectively, where $\delta_{j k}$ is Kronecker's delta.

Let $\left\{f_{j}\right\}_{j=1,2, \cdots, 6}$ denote the standard basis of $\mathbb{R}^{6}$. We define vector space isomorphisms, $\lambda^{-}: \mathbb{R}^{6} \rightarrow \mathcal{G}^{-}$and $\lambda^{+}: \mathbb{R}^{6} \rightarrow \mathcal{G}^{+}$, by

$$
\begin{aligned}
& \lambda^{-}\left(f_{j}\right):=-\frac{1}{2} b_{j}^{-}, \quad j=1,2, \cdots, 6, \\
& \lambda^{+}\left(f_{j}\right):=-b_{j}^{+}, \quad j=1,2, \cdots, 6
\end{aligned}
$$

respectively. Then, the isomorphisms $\lambda^{\mp}$ give rise to product structures $\wedge^{\mp}$ on $\mathbb{R}^{6}$ through

$$
\left[\lambda^{\mp}(x), \lambda^{\mp}(y)\right]=\lambda^{\mp}\left(x \wedge^{\mp} y\right), \quad x, y \in \mathbb{R}^{6},
$$

which means that both of the $\lambda^{\mp}$ are Lie algebra isomorphisms; $\left(\mathcal{G}^{\mp},[],\right) \cong$ $\left(\mathbb{R}^{6}, \wedge^{\mp}\right)$. 
To describe the wedge products $\wedge^{\mp}$ in a convenient manner, we regard $\mathbb{R}^{6}$ as the direct product $\mathbb{R}^{3} \times \mathbb{R}^{3}$ and express an element $x$ of $\mathbb{R}^{6}$ as

$$
x=\left(\begin{array}{l}
\boldsymbol{x}_{1} \\
\boldsymbol{x}_{2}
\end{array}\right) \in \mathbb{R}^{6}, \quad \text { with } \quad \boldsymbol{x}_{1}, \boldsymbol{x}_{2} \in \mathbb{R}^{3} .
$$

It is straightforward to show the following proposition.

Proposition 5.1 The exterior products $\wedge^{\mp}$ defined on $\mathbb{R}^{6}$ by (72) are expressed as

$$
\begin{aligned}
& \left(\begin{array}{l}
\boldsymbol{x}_{1} \\
\boldsymbol{x}_{2}
\end{array}\right) \wedge^{-}\left(\begin{array}{l}
\boldsymbol{y}_{1} \\
\boldsymbol{y}_{2}
\end{array}\right):=\left(\begin{array}{l}
\boldsymbol{x}_{1} \times \boldsymbol{y}_{1} \\
\boldsymbol{x}_{2} \times \boldsymbol{y}_{2}
\end{array}\right), \\
& \left(\begin{array}{l}
\boldsymbol{x}_{1} \\
\boldsymbol{x}_{2}
\end{array}\right) \wedge^{+}\left(\begin{array}{l}
\boldsymbol{y}_{1} \\
\boldsymbol{y}_{2}
\end{array}\right):=\left(\begin{array}{l}
\boldsymbol{x}_{1} \times \boldsymbol{y}_{1}-\boldsymbol{x}_{2} \times \boldsymbol{y}_{2} \\
\boldsymbol{x}_{1} \times \boldsymbol{y}_{2}+\boldsymbol{x}_{2} \times \boldsymbol{y}_{1}
\end{array}\right),
\end{aligned}
$$

respectively, where $\boldsymbol{x}_{j}, \boldsymbol{y}_{j} \in \mathbb{R}^{3}, j=1,2$, and $\times$ denotes the standard vector product on $\mathbb{R}^{3}$.

The $\left(\mathbb{R}^{6}, \wedge^{\mp}\right)$ are endowed also with the inner products $\Gamma^{\mp}$ by

$$
\Gamma^{\mp}(x, y):=\gamma^{\mp}\left(\lambda^{\mp}(x), \lambda^{\mp}(y)\right) .
$$

The inner products $\Gamma^{\mp}$ are shown to be explicitly expressed as

$$
\Gamma^{-}(x, y)=\frac{1}{4}\langle x, y\rangle, \quad \Gamma^{+}(x, y)=\frac{1}{2}\langle x, y\rangle,
$$

respectively, where $\langle$,$\rangle denotes the standard inner product on \mathbb{R}^{6}$. Thus, we have verified the following proposition.

Proposition 5.2 The $\lambda^{\mp}$ are isometries as well as Lie algebra isomorphisms;

$$
\left(\mathbb{R}^{6}, \wedge^{\mp}, \Gamma^{\mp}\right) \cong\left(\mathcal{G}^{\mp},[,], \gamma^{\mp}\right)
$$

We are now in a position to formulate Poisson structures on $\mathbb{R}^{6}$. In what follows, we use the symbol $q$ rather than $x$ to denote the variable in $\mathbb{R}^{6}$. The Poisson brackets $\{,\}^{\mp}$ are defined for functions on $\mathbb{R}^{6}$ through

$$
\begin{aligned}
& \{F, G\}^{-}(q):=16 \Gamma^{-}\left(q, \nabla F(q) \wedge^{-} \nabla G(q)\right), \\
& \{F, G\}^{+}(q):=4 \Gamma^{+}\left(q, \nabla F(q) \wedge^{+} \nabla G(q)\right),
\end{aligned}
$$

respectively, where $F$ and $G$ are functions of $q \in \mathbb{R}^{6}$ and where $\nabla$ denotes the standard gradient operator. 
The Poisson tensors associated with the Poisson structures $\{,\}^{\mp}$ are given by

$$
B_{i j}^{\mp}(q)=\left\{q_{i}, q_{j}\right\}^{\mp}, \quad q=\left(q_{j}\right) \in \mathbb{R}^{6}, \quad i, j=1, \cdots, 6,
$$

and can be written out as

$$
B^{-}(q)=4\left(\begin{array}{cc}
X_{1}(q) & 0 \\
0 & X_{2}(q)
\end{array}\right), \quad B^{+}(q)=2\left(\begin{array}{cc}
X_{1}(q) & X_{2}(q) \\
X_{2}(q) & -X_{1}(q)
\end{array}\right),
$$

where $X_{1}(q)$ and $X_{2}(q)$ are $3 \times 3$ matrices defined by

$$
X_{1}(q)=\left(\begin{array}{ccc}
0 & q_{3} & -q_{2} \\
-q_{3} & 0 & q_{1} \\
q_{2} & -q_{1} & 0
\end{array}\right), \quad X_{2}(q)=\left(\begin{array}{ccc}
0 & q_{6} & -q_{5} \\
-q_{6} & 0 & q_{4} \\
q_{5} & -q_{4} & 0
\end{array}\right),
$$

respectively. If $\left(q_{1}, q_{2}, q_{3}\right) \neq 0$ and $\left(q_{4}, q_{5}, q_{6}\right) \neq 0$, then the rank of $B^{-}(q)$ is four. If $q \neq 0$, then the rank of $B^{+}(q)$ is four. This fact suggests us that there are two Casimir functions with respect to the Poisson structures $\{,\}^{\mp}$, respectively.

Proposition 5.3 Let $C_{j}^{\mp}, j=1,2$, be functions defined on $\mathbb{R}^{6}$ by

$$
\begin{aligned}
& C_{1}^{-}(q):=\left\langle\boldsymbol{q}_{1}, \boldsymbol{q}_{1}\right\rangle, \quad C_{2}^{-}(q):=\left\langle\boldsymbol{q}_{2}, \boldsymbol{q}_{2}\right\rangle, \\
& C_{1}^{+}(q):=\left\langle\boldsymbol{q}_{1}, \boldsymbol{q}_{1}\right\rangle-\left\langle\boldsymbol{q}_{2}, \boldsymbol{q}_{2}\right\rangle, \quad C_{2}^{+}(q):=\left\langle\boldsymbol{q}_{1}, \boldsymbol{q}_{2}\right\rangle,
\end{aligned}
$$

respectively, where $q=\left(\begin{array}{l}\boldsymbol{q}_{1} \\ \boldsymbol{q}_{2}\end{array}\right) \in \mathbb{R}^{6}$. Then $C_{j}^{-}$and $C_{j}^{+}, j=1,2$, are Casimir functions of the Poisson structures $\{,\}^{-}$and $\{,\}^{+}$, respectively.

We omit the proof, which is performed by a straightforward calculation.

\subsection{Symplectic leaves of $\mathbb{R}^{6}$}

We shall show that the Poisson submanifolds determined by the Casimir functions $C_{j}^{\mp}$ can be identified with the (co-)adjoint orbits $i \mathcal{O}^{\mp}$ endowed with the KKS-forms $\Omega^{\mp}$. Choosing suitable constants, we define two Poisson submanifolds $\tilde{M}_{\mu, \kappa}^{\mp}$ of $\mathbb{R}^{6}$ to be

$$
\begin{aligned}
& \tilde{M}_{\mu, \kappa}^{-}:=\left\{q \in \mathbb{R}^{6} \mid C_{1}^{-}(q)=\chi_{1}^{-}(\mu, \kappa), C_{2}^{-}(q)=\chi_{2}^{-}(\mu, \kappa)\right\}, \\
& \tilde{M}_{\mu, \kappa}^{+}:=\left\{q \in \mathbb{R}^{6} \mid C_{1}^{+}(q)=\chi_{1}^{+}(\mu, \kappa), C_{2}^{+}(q)=\chi_{2}^{+}(\mu, \kappa)\right\},
\end{aligned}
$$

respectively, where $\chi_{j}^{\mp}(\mu, \kappa), j=1,2$, are constants given by

$$
\begin{array}{ll}
\chi_{1}^{-}(\mu, \kappa):=(\mu+4 \kappa)^{2}, & \chi_{2}^{-}(\mu, \kappa):=(\mu-4 \kappa)^{2}, \\
\chi_{1}^{+}(\mu, \kappa):=\mu^{2}-16 \kappa^{2}, & \chi_{2}^{+}(\mu, \kappa):=-4 \mu \kappa,
\end{array}
$$


respectively.

We here mention the elementary geometry of the submanifolds $\tilde{M}_{\mu, \kappa}^{\mp}$. From (81a) and (82a), it follows that $\tilde{M}_{\mu, \kappa}^{-}$is diffeomorphic with $S^{2}(|\mu+4 \kappa|) \times S^{2}(\mid \mu-$ $4 \kappa \mid)$, where $S^{2}(r)$ stands for the two-sphere of radius $r$. We have to note here that the radii $|\mu \pm 4 \kappa|$ may vanish, which never occurs for the Kepler problem case with $\mu=0$. To look into $\tilde{M}_{\mu, \kappa}^{+}$, we introduce a complex vector by $\boldsymbol{q}_{1}+i \boldsymbol{q}_{2}$ and a complex inner product on $\mathbb{C}^{3}$ in such a manner that $\boldsymbol{z} \cdot \boldsymbol{w}=\sum z_{j} w_{j}$. Then, the defining equations (81b) and (82b) are put together to give $\left(\boldsymbol{q}_{1}+i \boldsymbol{q}_{2}\right)^{2}=(\mu-4 i \kappa)^{2}$. This equation implies that $\tilde{M}_{\mu, \kappa}^{+}$is the complex two-sphere which is diffeomorphic with the tangent bundle of the (real) two-sphere, $T S^{2}$.

Proposition 5.4 The submanifolds $\tilde{M}_{\mu, \kappa}^{\mp}$ defined by (82) are diffeomorphic with the iso-energetic orbit spaces $M_{\mu, \kappa}^{\mp}$ defined by (37b) and by (51b), respectively.

Proof We show that $\mathcal{O}^{\mp}$ are immersed into $\tilde{M}_{\mu, \kappa}^{\mp}$, respectively. In the case of $\mathcal{G}^{-}$, for any point $i p \in i \mathcal{O}^{-} \subset \mathcal{G}^{-}$, there exists a point $w \in A_{1}^{-1}(4 \kappa) \cap \Phi^{-1}(\mu)$ such that

$$
\begin{aligned}
i p=i K^{-}(w) & =\sum_{j=1}^{3} J_{j+1}(w) i e_{j+1}+\sum_{j=1}^{3} J_{j+4}(w) i e_{j+4} \\
& =-2 \sum_{j=1}^{3} J_{j+1}(w) \lambda^{-}\left(f_{j}\right)-2 \sum_{j=1}^{3} J_{j+4}(w) \lambda^{-}\left(f_{j+3}\right) .
\end{aligned}
$$

Similarly, in the case of $\mathcal{G}^{+}$, for any point ip $\in i \mathcal{O}^{+} \subset \mathcal{G}^{+}$, there exists a point $w \in R_{1}^{-1}(4 \kappa) \cap \Phi^{-1}(\mu)$ such that

$$
\begin{aligned}
i p=i K^{+}(w) & =\frac{1}{2} \sum_{j=1}^{3}\left(J_{j+1}(w)+J_{j+4}(w)\right) i\left(e_{j+1}+e_{j+4}\right)+2 \sum_{j=1}^{3} J_{j+8}(w) i e_{j+8} \\
& =-\sum_{j=1}^{3}\left(J_{j+1}(w)+J_{j+4}(w)\right) \lambda^{+}\left(f_{j}\right)-2 \sum_{j=1}^{3} J_{j+8}(w) \lambda^{+}\left(f_{j+3}\right) .
\end{aligned}
$$

Since $J_{j+1}(w)$ and $J_{j+4}(w)$ are subject to the constraints (35), and $J_{j+1}(w)+$ $J_{j+4}(w)$ and $2 J_{j+8}(w)$ subject to $(36)$, the $i K^{-}(w)$ and the $i K^{+}(w)$ are mapped by $\left(\lambda^{-}\right)^{-1}$ and $\left(\lambda^{+}\right)^{-1}$ to $\tilde{M}_{\mu, \kappa}^{-}$and $\tilde{M}_{\mu, \kappa}^{+}$, respectively, on account of the fact that $\Phi=2 J_{0}=\mu, A_{1}=2 J_{1}=4 \kappa$, and $R_{1}=-2 J_{8}=4 \kappa$ on $A_{1}^{-1}(4 \kappa) \cap \Phi^{-1}(\mu)$ and $R_{1}^{-1}(4 \kappa) \cap \Phi^{-1}(\mu)$, respectively. Further, because of the invariance of $K^{-}$, (43b), for $U(1) \times U(1)$ and of $K^{+},(57 \mathrm{~b})$, for $U(1) \times \mathbb{R}$, the maps $\left(\lambda^{\mp}\right)^{-1} \circ i K^{\mp}$ project 
to respective injective maps $\left(\lambda^{\mp}\right)^{-1} \circ i \tilde{K}^{\mp}: M_{\mu, \kappa}^{-} \rightarrow \tilde{M}_{\mu, \kappa}^{\mp}$, where $\tilde{K}^{\mp}$ are defined in (48) and (62), respectively.

Conversely, since the $\tilde{M}_{\mu, \kappa}^{-}$is diffeomorphic to $S^{2}(|\mu+4 \kappa|) \times S^{2}(\mu-4 \kappa \mid)$, as is already stated, and then viewed as a (co-)adjoint orbit of $S U(2) \times S U(2)$, we can conclude that $\tilde{M}_{\mu, \kappa}^{-}$and $M_{\mu, \kappa}^{-}$are diffeomorphic to each other. To verify the diffeomorphism of $\tilde{M}_{\mu, \kappa}^{+}$with $M_{\mu, \kappa}^{+}$, we introduce a map of $\mathbb{C}^{3}$ into $\in \mathfrak{s l}(2, \mathbb{C})$ by

$$
\boldsymbol{z}=\left(z_{j}\right) \in \mathbb{C}^{3} \mapsto \sum_{j=1}^{3} z_{j} \sigma_{j} \in \mathfrak{s l}(2, \mathbb{C}),
$$

where $\sigma_{j}$ denote the Pauli spin matrices. Then, all the (co-)adjoint orbits of $S L(2, \mathbb{C})$ are known to be given by $\operatorname{det}\left(\sum_{j=1}^{3} z_{j} \sigma_{j}\right)=-\sum_{j} z_{j}^{2}=$ const. (We will make a remark on this mapping in the concluding section.) On setting $\boldsymbol{z}=$ $\boldsymbol{q}_{1}+i \boldsymbol{q}_{2}$, the defining equations $\left(\boldsymbol{q}_{1}+i \boldsymbol{q}_{2}\right)^{2}=(\mu-4 i \kappa)^{2}$ of $\tilde{M}_{\mu, \kappa}^{+}$is put in the form $\operatorname{det}\left(\sum_{j=1}^{3} z_{j} \sigma_{j}\right)=-(\mu-4 i \kappa)^{2}$. Thus, we see that $\tilde{M}_{\mu, \kappa}^{+}$and $M_{\mu, \kappa}^{+}$are diffeomorphic to each other as (co-)adjoint orbits of $S L(2, \mathbb{C})$. This ends the proof.

We study the symplectic geometry of $\tilde{M}_{\mu, \kappa}^{\mp}$. By the definition of $\tilde{M}_{\mu, \kappa}^{\mp}$, the rank of $\{,\}^{\mp}$ is four at any point on $\tilde{M}_{\mu, \kappa}^{\mp}$ if $|\mu| \neq 4 \kappa$. By the symplectic stratification theorem [2, pp. 302], if $|\mu| \neq 4 \kappa$, then $\tilde{M}_{\mu, \kappa}^{-}$and $\tilde{M}_{\mu, \kappa}^{+}$are symplectic leaves in $\mathbb{R}^{6}$, respectively, and $\left(\tilde{M}_{\mu, \kappa}^{\mp},\left.\{,\}^{\mp}\right|_{\tilde{M}_{\mu, \kappa}^{\mp}}\right)$ are symplectic manifolds, where $\left.\{,\}^{\mp}\right|_{\tilde{M}_{\mu, \kappa}^{\mp}}$ denote the restriction of the Poisson structures $\{,\}^{\mp}$ to $\tilde{M}_{\mu, \kappa}^{\mp}$, respectively.

We proceed to express symplectic forms to be defined naturally on the manifolds $\tilde{M}_{\mu, \kappa}^{\mp}$. As is easily verified, the tangent spaces to $\tilde{M}_{\mu, \kappa}^{\mp}$ at $q=\left(\begin{array}{l}\boldsymbol{q}_{1} \\ \boldsymbol{q}_{2}\end{array}\right) \in \tilde{M}_{\mu}^{\mp}$ are given by

$$
\begin{aligned}
& T_{q} \tilde{M}_{\mu, \kappa}^{-}=\left\{\left(\begin{array}{c}
\boldsymbol{v}_{1} \\
\boldsymbol{v}_{2}
\end{array}\right) \in \mathbb{R}^{6} \mid\left\langle\boldsymbol{v}_{1}, \boldsymbol{q}_{1}\right\rangle=0,\left\langle\boldsymbol{v}_{2}, \boldsymbol{q}_{2}\right\rangle=0\right\}, \\
& T_{q} \tilde{M}_{\mu, \kappa}^{+}=\left\{\left(\begin{array}{c}
\boldsymbol{v}_{1} \\
\boldsymbol{v}_{2}
\end{array}\right) \in \mathbb{R}^{6} \mid\left\langle\boldsymbol{v}_{1}, \boldsymbol{q}_{1}\right\rangle-\left\langle\boldsymbol{v}_{2}, \boldsymbol{q}_{2}\right\rangle=0,\left\langle\boldsymbol{v}_{1}, \boldsymbol{q}_{2}\right\rangle+\left\langle\boldsymbol{v}_{2}, \boldsymbol{q}_{1}\right\rangle=0\right\},
\end{aligned}
$$

respectively. As for tangent vectors, we give the following proposition without describing the proof, since it runs in a straightforward manner.

Proposition 5.5 For an arbitrary $a=\left(\begin{array}{c}\boldsymbol{a}_{1} \\ \boldsymbol{a}_{2}\end{array}\right) \in \mathbb{R}^{6}$ and $q=\left(\begin{array}{l}\boldsymbol{q}_{1} \\ \boldsymbol{q}_{2}\end{array}\right) \in \tilde{M}_{\mu, \kappa}^{\mp}$, the exterior products $a \wedge^{\mp} q$ are tangent vectors to $\tilde{M}_{\mu, \kappa}^{\mp}$ at $q$, respectively. Conversely, any tangent vectors to $\tilde{M}_{\mu, \kappa}^{\mp}$ at $q$ are put in the form $a \wedge^{\mp} q$, respectively. 
In order to describe the symplectic structures naturally defined on $\tilde{M}_{\mu, \kappa}^{\mp}$, we introduce the operator $\dagger: \mathbb{R}^{6} \rightarrow \mathbb{R}^{6}$ by

$$
\left(\begin{array}{l}
\boldsymbol{x}_{1} \\
\boldsymbol{x}_{2}
\end{array}\right)^{\dagger}:=\left(\begin{array}{c}
\boldsymbol{x}_{1} \\
-\boldsymbol{x}_{2}
\end{array}\right) \text {. }
$$

It is straightforward to show the following.

Proposition 5.6 For arbitrary $x, y, z \in \mathbb{R}^{6}$, we have

$$
\begin{aligned}
& \left(x^{\dagger}\right)^{\dagger}=x \\
& x^{\dagger} \wedge^{-} y^{\dagger}=x \wedge^{-} y, \quad x^{\dagger} \wedge^{+} y^{\dagger}=\left(x \wedge^{+} y\right)^{\dagger}, \\
& \Gamma^{-}\left(x \wedge^{-} y, z\right)=\Gamma^{-}\left(x, y \wedge^{-} z\right), \quad \Gamma^{+}\left(x \wedge^{+} y, z\right)=\Gamma^{+}\left(x, y^{\dagger} \wedge^{+} z\right), \\
& \left(\lambda^{-}(x)\right)^{*}=-\lambda^{-}(x), \quad\left(\lambda^{+}(x)\right)^{*}=-\lambda^{+}\left(x^{\dagger}\right),
\end{aligned}
$$

where the superscript $*$ indicates the Hermitian conjugate.

We proceed to the symplectic forms $\omega_{R}^{\mp}$ defined on $\tilde{M}_{\mu, \kappa}^{\mp}$ by

$$
\omega_{R}^{\mp}:=\left(\lambda^{\mp}\right)^{*} \Omega^{\mp},
$$

respectively, where $\Omega^{\mp}$ are the KKS-forms given in Eq.(65).

Proposition 5.7 For the tangent vectors $\tilde{\xi} \wedge^{\mp} \tilde{\nu}$ and $\tilde{\eta} \wedge^{\mp} \tilde{\nu}$ to $\tilde{M}_{\mu, \kappa}^{\mp}$ at $\tilde{\nu} \in \tilde{M}_{\mu, \kappa}^{\mp}$ with $\tilde{\xi}, \tilde{\eta} \in \mathbb{R}^{6}$, the symplectic forms $\omega_{R}^{\mp}$ are evaluated and expressed as

$$
\begin{aligned}
& \omega_{R}^{-}\left(\tilde{\xi} \wedge^{-} \tilde{\nu}, \tilde{\eta} \wedge^{-} \tilde{\nu}\right)(\tilde{\nu})=-\Gamma^{-}\left(\tilde{\nu}, \tilde{\xi} \wedge^{-} \tilde{\eta}\right), \\
& \omega_{R}^{+}\left(\tilde{\xi} \wedge^{+} \tilde{\nu}, \tilde{\eta} \wedge^{+} \tilde{\nu}\right)(\tilde{\nu})=-\Gamma^{+}\left(\tilde{\nu}, \tilde{\xi}^{\dagger} \wedge^{+} \tilde{\eta}^{\dagger}\right),
\end{aligned}
$$

respectively.

Proof Let $\xi=\lambda^{\mp}(\tilde{\xi}), \eta=\lambda^{\mp}(\tilde{\eta})$, and $\nu=\lambda^{\mp}(\tilde{\nu})$. Since $\lambda^{\mp}$ are Lie algebra isomorphisms, the left-hand sides of (90) and the definition (89) are put together to be brought into

$$
\omega_{R}^{\mp}\left(\tilde{\xi} \wedge^{\mp} \tilde{\nu}, \tilde{\eta} \wedge^{\mp} \tilde{\nu}\right)(\tilde{\nu})=\Omega^{\mp}([\xi, \nu],[\eta, \nu])(\nu) .
$$

We note here that

$$
\xi_{Q}(\nu)=\left\{\begin{array}{lll}
{[\xi, \nu]} & \text { for } & Q=\mathcal{G}^{-} \\
{\left[-\xi^{*}, \nu\right]} & \text { for } & Q=\mathcal{G}^{+}
\end{array}\right.
$$


Then, from the definitions (65) and (74), the right-hand side of Eq.(91) becomes

$$
\begin{aligned}
& \Omega^{-}([\xi, \nu],[\eta, \nu])(\nu)=-\Gamma^{-}\left(\tilde{\nu}, \tilde{\xi} \wedge^{-} \tilde{\eta}\right), \\
& \Omega^{+}([\xi, \nu],[\eta, \nu])(\nu)=-\Gamma^{+}\left(\tilde{\nu}, \tilde{\xi}^{\dagger} \wedge^{+} \tilde{\eta}^{\dagger}\right),
\end{aligned}
$$

respectively. This ends the proof.

Now we are in a position to describe Hamiltonian vector fields with respect to the symplectic forms $\omega_{R}^{\mp}$ on $\tilde{M}_{\mu, \kappa}^{\mp}$. Let $h$ be a Hamiltonian on $\tilde{M}_{\mu, \kappa}^{\mp}$. We will explicitly write out the Hamiltonian vector fields $X_{h}^{\mp}$ defined on $\tilde{M}_{\mu, \kappa}^{\mp}$ through

$$
\left.X_{h}^{\mp}\right\lrcorner \omega_{R}^{\mp}=-d h .
$$

Owing to Prop.5.5, we can put $X_{h}^{\mp}(q)$ in the form $X_{h}^{\mp}(q)=A^{\mp} \wedge^{\mp} q$ with $A^{\mp} \in \mathbb{R}^{6}$. Though $A^{-}$and $A^{+}$are different from each other, we denote $A^{\mp}$ by $A$ below for simplicity. Let $H: \mathbb{R}^{6} \rightarrow \mathbb{R}$ be a smooth extension of the function $h$ on $\tilde{M}_{\mu, \kappa}^{\mp}$. For an arbitrary $B \in \mathbb{R}^{6}$, we have

$$
\begin{aligned}
\Gamma^{-}\left(4 \nabla H(q) \wedge^{-} q, B\right) & =-\Gamma^{-}\left(4 \nabla H(q), B \wedge^{-} q\right)=-\left\langle\nabla H, B \wedge^{-} q\right\rangle \\
& =-d H\left(B \wedge^{-} q\right)=-d h\left(B \wedge^{-} q\right) \\
& =\omega_{R}^{-}\left(X_{h}^{-}(q), B \wedge^{-} q\right)=\omega_{R}^{-}\left(A \wedge^{-} q, B \wedge^{-} q\right) \\
& =-\Gamma^{-}\left(q, A \wedge^{-} B\right) \\
& =\Gamma^{-}\left(A \wedge^{-} q, B\right),
\end{aligned}
$$

where $\nabla$ denotes the usual gradient operator for functions on $\mathbb{R}^{6}$, and where we have used the fact that $d H\left(B \wedge^{\mp} q\right)=d h\left(B \wedge^{\mp} q\right)$, which is easily verified. In a similar manner, we have

$$
\begin{aligned}
\Gamma^{+}\left(2 \nabla H(q) \wedge^{+} q^{\dagger}, B\right) & =-\Gamma^{+}\left(2 \nabla H(q), B \wedge^{+} q\right)=-\left\langle\nabla H, B \wedge^{+} q\right\rangle \\
& =-d H\left(B \wedge^{+} q\right)=-d h\left(B \wedge^{+} q\right) \\
& =\omega_{R}^{+}\left(X_{h}^{+}(q), B \wedge^{+} q\right)=\omega_{R}^{+}\left(A \wedge^{+} q, B \wedge^{+} q\right) \\
& =-\Gamma^{+}\left(q, A^{\dagger} \wedge^{+} B^{\dagger}\right) \\
& =\Gamma^{+}\left(A^{\dagger} \wedge^{+} q^{\dagger}, B\right) .
\end{aligned}
$$

Comparing both sides of Eqs.(93) and of (94), we obtain the Hamiltonian vector fields $X_{h}^{\mp}$, which are given in the following proposition. 
Proposition 5.8 For a Hamiltonian $h$ on $\tilde{M}_{\mu, \kappa}^{\mp}$, the associated Hamiltonian vector fields $X_{h}^{\mp}$ take the form

$$
\begin{aligned}
& X_{h}^{-}(q)=4 \nabla H(q) \wedge^{-} q, \quad q \in \tilde{M}_{\mu, \kappa}^{-}, \\
& X_{h}^{+}(q)=2(\nabla H(q))^{\dagger} \wedge^{+} q, \quad q \in \tilde{M}_{\mu, \kappa}^{+},
\end{aligned}
$$

respectively, where $H$ is a smooth extension of $h$.

In terms of Hamiltonian vector fields, Poisson brackets $\{,\}_{\tilde{M}_{\mu, \kappa}^{\mp}}$ on $\left(\tilde{M}_{\mu, \kappa}^{\mp}, \omega_{R}^{\mp}\right)$ are defined, as usual, by

$$
\{f, g\}_{\tilde{M}_{\mu, \kappa}^{\mp}}(q):=-\omega_{R}^{\mp}\left(X_{f}, X_{g}\right), \quad q \in \tilde{M}_{\mu, \kappa}^{\mp},
$$

where $X_{f}$ and $X_{g}$ are the Hamiltonian vector fields associated with functions $f$ and $g$ on $\tilde{M}_{\mu, \kappa}^{\mp}$, respectively; $\left.\left.X_{f}\right\rfloor \omega_{R}^{\mp}=-d f, X_{g}\right\rfloor \omega_{R}^{\mp}=-d g$.

So far we have obtained two Poisson brackets on each of $\tilde{M}_{\mu, \kappa}^{\mp}$; one is $\{,\}_{\tilde{M}_{\mu, \kappa}^{\mp}}$ and the other, $\left.\{,\}^{\mp}\right|_{\tilde{M}_{\mu, \kappa}^{\mp}}$, the restriction of $\{,\}^{\mp}$ to $\tilde{M}_{\mu, \kappa}^{\mp}$. However, these Poisson brackets are the same, since we can easily verify, by using Prop. 5.8, that

$$
\left.\{F, G\}^{\mp}\right|_{\tilde{M}_{\mu, \kappa}^{\mp}}=\{f, g\}_{\tilde{M}_{\mu, \kappa}^{\mp}},
$$

where $f=\left.F\right|_{\tilde{M}_{\mu, \kappa}^{\mp}}$ and $g=\left.G\right|_{\tilde{M}_{\mu, \kappa}^{\mp}}$, respectively. We state this fact in the form of a theorem.

Theorem 5.9 The Poisson submanifolds $\left(\tilde{M}_{\mu, \kappa}^{\mp},\left.\{,\}^{\mp}\right|_{\tilde{M}_{\mu, \kappa}^{\mp}}\right)$ are symplectomorphic to $\left(\tilde{M}_{\mu, \kappa}^{\mp},\{,\}_{\tilde{M}_{\mu, \kappa}^{\mp}}^{\mp}\right)$, respectively.

In terms of the Hamiltonian vector fields (95), Hamilton's equations of motion on $\tilde{M}_{\mu, \kappa}^{\mp}$ are put in the form

$$
\begin{aligned}
& \frac{d q}{d t}=4 \nabla H(q) \wedge^{-} q, \quad q \in \tilde{M}_{\mu, \kappa}^{-}, \\
& \frac{d q}{d t}=2(\nabla H(q))^{\dagger} \wedge^{+} q, \quad q \in \tilde{M}_{\mu, \kappa}^{+}
\end{aligned}
$$

respectively, where $H$ is an extension of the Hamiltonian $h$ on $\tilde{M}_{\mu, \kappa}^{\mp}$. These equations of motion can be written out as follows:

Proposition 5.10 Hamilton's equations of motion on the symplectic leaves $\tilde{M}_{\mu, \kappa}^{\mp}$ 
are expressed as

$$
\begin{aligned}
& \frac{d}{d t}\left(\begin{array}{l}
\boldsymbol{q}_{1} \\
\boldsymbol{q}_{2}
\end{array}\right)=4\left(\begin{array}{l}
\nabla_{\boldsymbol{q}_{1}} H \times \boldsymbol{q}_{1} \\
\nabla_{\boldsymbol{q}_{2}} H \times \boldsymbol{q}_{2}
\end{array}\right), \quad q=\left(\begin{array}{l}
\boldsymbol{q}_{1} \\
\boldsymbol{q}_{2}
\end{array}\right) \in \tilde{M}_{\mu, \kappa}^{-}, \\
& \frac{d}{d t}\left(\begin{array}{l}
\boldsymbol{q}_{1} \\
\boldsymbol{q}_{2}
\end{array}\right)=2\left(\begin{array}{c}
\nabla_{\boldsymbol{q}_{1}} H \times \boldsymbol{q}_{1}+\nabla_{\boldsymbol{q}_{2}} H \times \boldsymbol{q}_{2} \\
\nabla_{\boldsymbol{q}_{1}} H \times \boldsymbol{q}_{2}-\nabla_{\boldsymbol{q}_{2}} H \times \boldsymbol{q}_{1}
\end{array}\right), \quad q=\left(\begin{array}{c}
\boldsymbol{q}_{1} \\
\boldsymbol{q}_{2}
\end{array}\right) \in \tilde{M}_{\mu, \kappa}^{+},
\end{aligned}
$$

where $\nabla_{\boldsymbol{q}_{1}}$ and $\nabla_{\boldsymbol{q}_{2}}$ are the usual gradient operators with respect to $\boldsymbol{q}_{1}$ and $\boldsymbol{q}_{2}$, respectively, and where $H$ is a smooth extension of a Hamiltonian on $\tilde{M}_{\mu, \kappa}^{\mp}$.

\subsection{Poisson mechanics on $\mathbb{R}^{6}$}

We show that Hamilton's equations of motion, (98) or (99), are put in the form of Poisson's equations of motion. Let $F: \mathbb{R}^{6} \rightarrow \mathbb{R}$ be a scalar-valued function and $\boldsymbol{G}: \mathbb{R}^{6} \rightarrow \mathbb{R}^{6}$ be a vector-valued function on $\mathbb{R}^{6}$, which is expressed as $\boldsymbol{G}(x)=\sum_{j=1}^{6} G_{j}(x) f_{j}$, where $G_{j}: \mathbb{R}^{6} \rightarrow \mathbb{R}, j=1,2, \cdots, 6$, are scalar-valued functions and where $\left\{f_{j}\right\}_{j=1,2, \cdots, 6}$ is the standard basis of $\mathbb{R}^{6}$. We can extend the definition of Poisson bracket in such a way that the Poisson bracket of $F$ and $\boldsymbol{G}$ is expressed as

$$
\{F, \boldsymbol{G}\}^{\mp}(x):=\sum_{j=1}^{6}\left\{F, G_{j}\right\}^{\mp}(x) f_{j} .
$$

Theorem 5.11 Let $F: \mathbb{R}^{6} \rightarrow \mathbb{R}$ be a function. Then, the vector-valued Poisson brackets of $F$ and $q \in \mathbb{R}^{6}$ are put in the form

$$
\begin{aligned}
& -\{F, q\}^{-}=4 \nabla F(q) \wedge^{-} q, \\
& -\{F, q\}^{+}=2(\nabla F(q))^{\dagger} \wedge^{+} q,
\end{aligned}
$$

respectively. If $q$ is restricted to $\tilde{M}_{\mu, \kappa}^{\mp}$ and if $F$ is a smooth extension of a function $f$ on $\tilde{M}_{\mu, \kappa}^{\mp}$, then one has

$$
-\{F, q\}^{\mp}=X_{f}^{\mp}(q) \quad \text { for } \quad q \in \tilde{M}_{\mu, \kappa}^{\mp} .
$$

Proof Let $\left\{\boldsymbol{e}_{j}\right\}_{j=1,2,3}$ be the standard basis of $\mathbb{R}^{3}$ and let $\langle$,$\rangle be the standard$ inner product on $\mathbb{R}^{3}$. By the definition (77a) along with $q=\left(q_{k}\right)=\left(\begin{array}{l}\boldsymbol{q}_{1} \\ \boldsymbol{q}_{2}\end{array}\right) \in \mathbb{R}^{6}$, the Poisson brackets $\left\{F, q_{k}\right\}^{-}, k=1,2, \cdots, 6$, are written out as

$$
\begin{aligned}
-\left\{F, q_{j}\right\}^{-}(q) & =4\left\langle\nabla_{\boldsymbol{q}_{1}} F(q) \times \boldsymbol{q}_{1}, \boldsymbol{e}_{j}\right\rangle=\left(4 \nabla F(q) \wedge^{-} q\right)_{j}, \\
-\left\{F, q_{j+3}\right\}^{-}(q) & =4\left\langle\nabla_{\boldsymbol{q}_{2}} F(q) \times \boldsymbol{q}_{2}, \boldsymbol{e}_{j}\right\rangle=\left(4 \nabla F(q) \wedge^{-} q\right)_{j+3},
\end{aligned}
$$


where $j=1,2,3$, and where $\nabla_{\boldsymbol{q}_{1}}$ and $\nabla_{\boldsymbol{q}_{2}}$ are the gradient operators with respect to $\boldsymbol{q}_{1}$ and $\boldsymbol{q}_{2}$, respectively. From Eqs.(103) and (104), we obtain for $q=\sum q_{k} f_{k}$

$$
-\{F, q\}^{-}(q)=-\sum_{k=1}^{6}\left\{F, q_{k}\right\}^{-}(q) f_{k}=4 \nabla F(q) \wedge^{-} q .
$$

As for the Poisson bracket $\{,\}^{+}$, the definition (77b) along with $q=\left(q_{k}\right)=$ $\left(\begin{array}{l}\boldsymbol{q}_{1} \\ \boldsymbol{q}_{2}\end{array}\right) \in \mathbb{R}^{6}$ yields the Poisson brackets between $F$ and $q_{k}, k=1,2, \cdots, 6$, in a similar manner as above,

$$
-\{F, q\}^{+}(q)=-\sum_{k=1}^{6}\left\{F, q_{k}\right\}^{+}(q) f_{k}=2(\nabla F(q))^{\dagger} \wedge^{+} q .
$$

Equations (102) are consequences from (101) together with (95). This ends the proof.

Eqs.(101a) and (101b) imply that the Hamiltonian dynamical systems on the symplectic manifolds $\left(\tilde{M}_{\mu, \kappa}^{\mp}, \omega_{R}^{\mp}\right)$ can be regarded as those on the Poisson manifolds $\left(\mathbb{R}^{6},\{,\}^{\mp}\right)$. Thus, the equations of motion (98) can be put in the form

$$
\frac{d q}{d t}=\{q, H\}^{\mp}, \quad q \in \mathbb{R}^{6}
$$

Here we have to note that since the Casimir functions $C_{j}^{\mp}$ given in (81) are constants of motion with respect to (105), the flow determined by (105) is naturally restricted on $\tilde{M}_{\mu, \kappa}^{\mp}$.

\subsection{Dynamics on the iso-energetic orbit spaces $M_{\mu, \kappa}^{\mp}$}

In view of the existence of the Casimir functions $C_{j}^{\mp}$, we may regard the equations of motion (99a) and (99b) as defined on $\mathbb{R}^{6}$ without the restriction to $\tilde{M}_{\mu, \kappa}^{\mp}$. With this in mind, we introduce new coordinates $\left(\boldsymbol{L}^{\mp}, \boldsymbol{K}^{\mp}\right)$ on $\mathbb{R}^{6}$ by

$$
\begin{aligned}
L_{j}^{-} & =\frac{1}{4}\left(q_{j+3}+q_{j}\right), \quad K_{j}^{-}=\frac{1}{4}\left(q_{j+3}-q_{j}\right), \quad j=1,2,3, \quad \text { for } \quad \mathcal{G}^{-}, \\
L_{j}^{+} & =\frac{1}{2} q_{j}, \quad K_{j}^{+}=\frac{1}{2} q_{j+3}, \quad j=1,2,3, \quad \text { for } \quad \mathcal{G}^{+},
\end{aligned}
$$

respectively. Then, the equations of motion (99a) and (99b) are rewritten in terms of $\boldsymbol{L}^{\mp}$ and $\boldsymbol{K}^{\mp}$ as

$$
\frac{d}{d t}\left(\begin{array}{c}
\boldsymbol{L}^{\mp} \\
\boldsymbol{K}^{\mp}
\end{array}\right)=\left(\begin{array}{c}
\nabla_{\boldsymbol{L}^{\mp}} H \times \boldsymbol{L}^{\mp}+\nabla_{\boldsymbol{K}^{\mp}} H \times \boldsymbol{K}^{\mp} \\
\nabla_{\boldsymbol{L}^{\mp}} H \times \boldsymbol{K}^{\mp} \pm \nabla_{\boldsymbol{K}^{\mp}} H \times \boldsymbol{L}^{\mp}
\end{array}\right),
$$


respectively, where $\nabla_{\boldsymbol{L}^{\mp}}$ and $\nabla_{\boldsymbol{K}^{\mp}}$ are the usual gradient operators with respect to $\boldsymbol{L}^{\mp}$ and $\boldsymbol{K}^{\mp}$, respectively. We note that only the signs of $\nabla_{\boldsymbol{K}^{\mp}} H \times \boldsymbol{L}^{\mp}$ in the lower row of the right-hand side of Eq. (107) distinguish the cases $\mathcal{G}^{\mp}$.

If $q \in \tilde{M}_{\mu, \kappa}^{\mp}$, then the constraint conditions in (82) require the $\boldsymbol{K}^{\mp}$ and $\boldsymbol{L}^{\mp}$ to be subject to the constraints

$$
\left\langle\boldsymbol{L}^{-}, \boldsymbol{L}^{-}\right\rangle+\left\langle\boldsymbol{K}^{-}, \boldsymbol{K}^{-}\right\rangle=\frac{1}{4} \mu^{2}+4 \kappa^{2}, \quad\left\langle\boldsymbol{L}^{-}, \boldsymbol{K}^{-}\right\rangle=\mu \kappa
$$

and

$$
\left\langle\boldsymbol{L}^{+}, \boldsymbol{L}^{+}\right\rangle-\left\langle\boldsymbol{K}^{+}, \boldsymbol{K}^{+}\right\rangle=\frac{1}{4} \mu^{2}-4 \kappa^{2}, \quad\left\langle\boldsymbol{L}^{+}, \boldsymbol{K}^{+}\right\rangle=-\mu \kappa,
$$

respectively. On the other hand, we can easily verify that the left-hand sides of (108) and (109), $\left\langle\boldsymbol{L}^{\mp}, \boldsymbol{L}^{\mp}\right\rangle \pm\left\langle\boldsymbol{K}^{\mp}, \boldsymbol{K}^{\mp}\right\rangle$ and $\left\langle\boldsymbol{L}^{\mp}, \boldsymbol{K}^{\mp}\right\rangle$, are constants of motion for the equations of motion (107).

In conclusion of this section, we show that the variables $\boldsymbol{K}^{\mp}$ and $\boldsymbol{L}^{\mp}$ are related with the constants of motion for the MIC-Kepler problem. If $q \in \tilde{M}_{\mu, \kappa}^{\mp}$, the $q_{k}, k=1, \cdots, 6$, can be expressed in terms of $J_{\ell}$ through (84) and (85), so that $L_{j}^{-}, K_{j}^{-}, j=1,2,3$, can be expressed also in $J_{\ell}$ and eventually in terms of $\left(x_{i}, y_{i}\right) \in \mathbb{R}^{4} \times \mathbb{R}^{4}$ as

$$
\begin{aligned}
L_{1}^{-}=-\frac{1}{2}\left(J_{2}+J_{5}\right) & =\frac{1}{2}\left(-x_{2} y_{1}+x_{1} y_{2}-x_{3} y_{4}+x_{4} y_{3}\right) \\
L_{2}^{-}=-\frac{1}{2}\left(J_{3}+J_{6}\right) & =\frac{1}{2}\left(-x_{3} y_{1}+x_{1} y_{3}-x_{4} y_{2}+x_{2} y_{4}\right) \\
L_{3}^{-}=-\frac{1}{2}\left(J_{4}+J_{7}\right) & =\frac{1}{2}\left(-x_{1} y_{4}+x_{4} y_{1}-x_{3} y_{2}+x_{2} y_{3}\right) \\
K_{1}^{-}=-\frac{1}{2}\left(J_{5}-J_{2}\right) & =\frac{1}{4}\left(-x_{1}^{2}-x_{2}^{2}+x_{3}^{2}+x_{4}^{2}\right)+\frac{1}{4}\left(-y_{1}^{2}-y_{2}^{2}+y_{3}^{2}+y_{4}^{2}\right), \\
K_{2}^{-}=-\frac{1}{2}\left(J_{6}-J_{3}\right) & =\frac{1}{2}\left(-x_{2} x_{3}+x_{1} x_{4}\right)+\frac{1}{2}\left(-y_{2} y_{3}+y_{1} y_{4}\right) \\
K_{3}^{-}=-\frac{1}{2}\left(J_{7}-J_{4}\right) & =\frac{1}{2}\left(x_{1} x_{3}+x_{2} x_{4}\right)+\frac{1}{2}\left(y_{1} y_{3}+y_{2} y_{4}\right) .
\end{aligned}
$$


In the same manner, $L_{k}^{+}, K_{j}^{+}, j=1,2,3$, are described as

$$
\begin{aligned}
L_{1}^{+} & =-\frac{1}{2}\left(J_{2}+J_{5}\right)=\frac{1}{2}\left(-x_{2} y_{1}+x_{1} y_{2}-x_{3} y_{4}+x_{4} y_{3}\right), \\
L_{2}^{+} & =-\frac{1}{2}\left(J_{3}+J_{6}\right)=\frac{1}{2}\left(-x_{3} y_{1}+x_{1} y_{3}-x_{4} y_{2}+x_{2} y_{4}\right), \\
L_{3}^{+} & =-\frac{1}{2}\left(J_{4}+J_{7}\right)=\frac{1}{2}\left(-x_{1} y_{4}+x_{4} y_{1}-x_{3} y_{2}+x_{2} y_{3}\right), \\
K_{1}^{+} & =-J_{9}=\frac{1}{4}\left(-x_{3}^{2}-x_{4}^{2}+x_{1}^{2}+x_{2}^{2}\right)+\frac{1}{4}\left(-y_{1}^{2}-y_{2}^{2}+y_{3}^{2}+y_{4}^{2}\right), \\
K_{2}^{+} & =-J_{10}=\frac{1}{2}\left(-x_{1} x_{4}+x_{2} x_{3}\right)+\frac{1}{2}\left(-y_{2} y_{3}+y_{1} y_{4}\right), \\
K_{3}^{+} & =-J_{11}=-\frac{1}{2}\left(x_{1} x_{3}+x_{2} x_{4}\right)+\frac{1}{2}\left(y_{1} y_{3}+y_{2} y_{4}\right) .
\end{aligned}
$$

We note here that $L_{j}^{-}=L_{j}^{+}, j=1,2,3$, if expressed in terms of $\left(x_{j}, y_{j}\right)$. If viewed as function of $\left(x_{j}, y_{j}\right)$, the $\boldsymbol{L}^{-}, \boldsymbol{K}^{-}$and $\boldsymbol{L}^{+}, \boldsymbol{K}^{+}$are constants of motion for the harmonic and the repulsive oscillators, respectively. The $\boldsymbol{K}^{\mp}$ and $\boldsymbol{L}^{\mp}$ project through $\pi_{\mu}$ to the angular momentum vector and the Laplace-Runge-Lenz vector, respectively, within constant multiples, if the coefficients $1 / 4$ and $1 / 2$ of the quadratic terms in $x_{k}$ are replaced by $-2 H_{c}$ and $-4 H_{c}$ in the $\boldsymbol{K}^{-}$and by $2 H_{c}$ and $4 H_{c}$ in the $\boldsymbol{K}^{+}$, respectively, before reduction procedure (see also $[4,6]$ ).

Thus, we have obtained the equations of motion on the iso-energetic orbits spaces at negative and positive energies for the MIC-Kepler problem.

Proposition 5.12 Since the iso-energetic orbit spaces $M_{\mu, \kappa}^{\mp}$ are diffeomorphic with $\tilde{M}_{\mu, \kappa}^{\mp}$, Eqs. (107) serve as the equations of motion on the iso-energetic orbit spaces $M_{\mu, \kappa}^{\mp}$. If $H$ is chosen suitably as a perturbed Hamiltonian, Eqs. (107) can be viewed as the equations of motion for perturbed MIC-Kepler problem of integrable type. The variables $\boldsymbol{L}^{\mp}, \boldsymbol{K}^{\mp}$ are related with the constants of motion for the MIC-Kepler problem, where $\boldsymbol{L}^{-}=\boldsymbol{L}^{+}$are the angular momentum and $\boldsymbol{K}^{\mp}$ the Laplace-Runge-Lenz vectors with negative and positive energies, respectively.

The equations of motion (107) written in terms of $\boldsymbol{L}^{-}$and $\boldsymbol{K}^{-}$are already known in [6]. In fact, if expressed in terms of $L_{j}^{-}, K_{j}^{-}$, the Poisson structure given in (78) and (79) takes the same form as that given in [6]. However, the equations expressed in terms of $\boldsymbol{L}^{+}$and $\boldsymbol{K}^{+}$are new. A point to make here is that the equations of motion in both cases are obtained on an equal footing. 


\section{Perturbation in normal form}

In this section, we discuss the perturbation of the harmonic and the repulsive oscillators on $\mathbb{R}^{4} \times \mathbb{R}^{4}$, which is related with the perturbation of the conformal Kepler problem and further with the perturbation of the MIC-Kepler problem if projected on the iso-energetic orbit spaces $M_{\mu, \kappa}^{\mp}$. Though the perturbation by small constant electric and magnetic fields which are not orthogonal to each other is already studied [7-11], the small electric and magnetic fields to be treated below are restricted to those orthogonal to each other for simplicity.

\subsection{A perturbed MIC-Kepler problem}

In the presence of constant magnetic and electric fields [4], the Hamiltonian of a perturbed MIC-Kepler problem is expressed as

$$
\begin{aligned}
H_{\mu}^{(\varepsilon)} & =H_{\mu}+N^{(\varepsilon)} \\
& =H_{\mu}+\boldsymbol{F} \cdot \boldsymbol{r}+\frac{1}{2}(\boldsymbol{G} \cdot(\boldsymbol{r} \times \boldsymbol{p}))+\frac{1}{8}(\boldsymbol{G} \times \boldsymbol{r})^{2},
\end{aligned}
$$

where $\boldsymbol{G}$ and $\boldsymbol{F}$ are the constant magnetic and electric field vectors orthogonal to each other, respectively, and where $\boldsymbol{r}=\left(r_{k}\right) \in \mathbb{R}^{3}$.

We take the Cartesian coordinates $\left(r_{k}\right)$ of $\mathbb{R}^{3}$ in such a manner that $\boldsymbol{G}$ and $\boldsymbol{F}$ are expressed as $\boldsymbol{G}=(G, 0,0)^{T}$ and $\boldsymbol{F}=(0, F, 0)^{T}$ with $G=O(\varepsilon)$ and $F=O(\varepsilon)$, where $\varepsilon$ is a small parameter. Then, the perturbation term given in (110) becomes

$$
N^{(\varepsilon)}=F r_{2}+\frac{1}{2} G\left(r_{2} p_{3}-r_{3} p_{2}\right)+\frac{1}{8} G^{2}\left(r_{2}^{2}+r_{3}^{2}\right) .
$$

In order to work with $H_{\mu}^{(\varepsilon)}$, we wish to view it as a reduced Hamiltonian from that for a perturbed conformal Kepler problem. To this end, we define a perturbation function $N_{c}^{(\varepsilon)}$ on $T^{*} \dot{\mathbb{R}}^{4}$ through

$$
\pi_{\mu}^{*} N^{(\varepsilon)}=\iota_{\mu}^{*} N_{c}^{(\varepsilon)}
$$

Then, we obtain a perturbed conformal Kepler problem $\left(T^{*} \dot{\mathbb{R}}^{4}, d \theta, H_{c}^{(\varepsilon)}\right)$, where

$$
H_{c}^{(\varepsilon)}=H_{c}+N_{c}^{(\varepsilon)} .
$$

In view of the relation (13) between the conformal Kepler problem and the harmonic and the repulsive oscillators, we define Hamiltonians for perturbed harmonic and repulsive oscillators to be

$$
A^{(\varepsilon)}=A_{1}+N_{A}^{(\varepsilon)}, \quad R^{(\varepsilon)}=R_{1}+N_{R}^{(\varepsilon)}
$$


respectively, where $A_{1}$ and $R_{1}$ are the harmonic and the repulsive oscillator Hamiltonians with $\lambda=1$, respectively, and where

$$
4 \rho N_{c}^{(\varepsilon)}=N_{A}^{(\varepsilon)}=N_{R}^{(\varepsilon)} .
$$

Then, Eqs.(13a) and (13b) with $\lambda=1$ are perturbed to be brought into

$$
\begin{aligned}
& 4 \rho\left(H_{c}^{(\varepsilon)}+\frac{1}{8}\right)=A^{(\varepsilon)}-4 \kappa, \\
& 4 \rho\left(H_{c}^{(\varepsilon)}-\frac{1}{8}\right)=R^{(\varepsilon)}-4 \kappa,
\end{aligned}
$$

respectively, where the perturbation of (13c) is outside our scope. Thus, according to the sign of the energy, the perturbed conformal Kepler problem is associated with the perturbed harmonic and repulsive oscillators. Further, a calculation shows that the perturbation term $N_{c}^{(\varepsilon)}$ multiplied by $4 \rho$ is put in the form

$$
\begin{aligned}
& 4 \rho N_{c}^{(\varepsilon)} \\
= & 8 F\left(x_{2} x_{3}-x_{1} x_{4}\right)\left(x_{1}^{2}+x_{2}^{2}+x_{3}^{2}+x_{4}^{2}\right) \\
& +2 G\left(x_{2} x_{3}-x_{1} x_{4}\right)\left(x_{1} y_{1}+x_{2} y_{2}-x_{3} y_{3}-x_{4} y_{4}\right) \\
& +G\left(x_{1}^{2}+x_{2}^{2}-x_{3}^{2}-x_{4}^{2}\right)\left(x_{1} y_{4}+y_{1} x_{4}-x_{2} y_{3}-y_{2} x_{3}\right) \\
& +\frac{1}{2} G^{2}\left(x_{1}^{2}+x_{2}^{2}+x_{3}^{2}+x_{4}^{2}\right)\left(\left(x_{1}^{2}+x_{2}^{2}-x_{3}^{2}-x_{4}^{2}\right)^{2}+4\left(x_{2} x_{3}-x_{1} x_{4}\right)^{2}\right) .
\end{aligned}
$$

We note that $4 \rho N_{c}^{(\varepsilon)}$ is a polynomial in $\left(x_{j}, y_{j}\right), j=1,2,3,4$, whose degree is greater than or equal to four, and further that it is invariant under the $S O(2)$ action (2). This implies that $4 \rho N_{c}^{(\varepsilon)}$ reduces to a function on $T^{*} \mathbb{R}^{3}$.

\subsection{Perturbed Hamiltonians in BG normal form}

We apply the method of the Birkhoff-Gustavson (BG) normal form [16-19] to the perturbed Hamiltonians (114). Though the method is originally applied to perturbed harmonic oscillators, it can be extended so as to be applicable to perturbed repulsive oscillators. By means of canonical transformations, $(x, y) \mapsto(\xi, \eta)$, we can transform the perturbed harmonic and repulsive oscillator Hamiltonians into those in BG-normal form up to degree $r$ in $(\xi, \eta)$ as follows [21-23]:

$$
\begin{aligned}
& \mathcal{A}^{(\varepsilon)}=\mathcal{A}+\mathcal{N}_{A}^{(\varepsilon)}=\frac{1}{2} \sum_{j=1}^{4}\left(\eta_{j}^{2}+\xi_{j}^{2}\right)+\sum_{k=3}^{r} G_{k}^{-}, \\
& \mathcal{R}^{(\varepsilon)}=\mathcal{R}+\mathcal{N}_{R}^{(\varepsilon)}=\frac{1}{2} \sum_{j=1}^{4}\left(\eta_{j}^{2}-\xi_{j}^{2}\right)+\sum_{k=3}^{r} G_{k}^{+},
\end{aligned}
$$


where

$$
\mathcal{A}=\frac{1}{2} \sum_{j=1}^{4}\left(\eta_{j}^{2}+\xi_{j}^{2}\right), \quad \mathcal{R}=\frac{1}{2} \sum_{j=1}^{4}\left(\eta_{j}^{2}-\xi_{j}^{2}\right),
$$

and where $G_{k}^{\mp}, k=3,4, \cdots, r$, are homogeneous polynomials of degree $k$ in $(\xi, \eta)$, which satisfy

$$
\left\{\Phi, G_{k}^{\mp}\right\}=0, \quad\left\{\frac{1}{2} \sum_{j=1}^{4}\left(\eta_{j}^{2} \pm \xi_{j}^{2}\right), G_{k}^{\mp}\right\}=0, \quad k=3,4, \cdots, r .
$$

We notice here that the first conditions $\left\{\Phi, G_{k}^{\mp}\right\}=0$ of the above equations are imposed so that the $G_{k}^{\mp}$ may be reduced by the $S O(2)$ symmetry. This is because $N_{A}^{(\varepsilon)}=N_{R}^{(\varepsilon)}$ is $S O(2)$ invariant. The second condition of (120) says that the perturbation terms are in normal form. These two conditions imply that the Hamiltonians $\mathcal{A}^{(\varepsilon)}$ and $\mathcal{R}^{(\varepsilon)}$ for the perturbed harmonic and the perturbed repulsive oscillators are invariant under $U(1) \times U(1)$ and $U(1) \times \mathbb{R}$, respectively, where $U(1) \times U(1)$ and $U(1) \times \mathbb{R}$ are the symmetry groups treated in Sec. 4 .

Suppose we have obtained $\mathcal{A}^{(\varepsilon)}$ and $\mathcal{R}^{(\varepsilon)}$ in BG-normal form. Then, because of the symmetry by $U(1) \times U(1)$ and $U(1) \times \mathbb{R}$, the perturbed Hamiltonians $\mathcal{A}^{(\varepsilon)}$ and $\mathcal{R}^{(\varepsilon)}$ can be reduced on the respective iso-energetic orbit spaces $M_{\mu, \kappa}^{\mp}\left(\right.$ or $\tilde{M}_{\mu, \kappa}^{\mp}$ ) and further expressed in terms of $J_{\ell}$. Put in detail, in the case of the perturbed harmonic oscillator, the BG normalized Hamiltonian $\mathcal{A}^{(\varepsilon)}(\xi, \eta)$ given in (118a) can be written in terms of $J_{j+1}(\xi, \eta), J_{j+4}(\xi, \eta)$, and in the case of the perturbed repulsive oscillator, the $\mathrm{BG}$ normalized Hamiltonian $\mathcal{R}^{(\varepsilon)}(\xi, \eta)$ given in (118b) can be written in terms of $J_{j+1}(\xi, \eta)+J_{j+4}(\xi, \eta), J_{j+8}(\xi, \eta), j=1,2,3$ (see (84) and $(85))$.

\subsection{Normal form calculation on Maxima}

Calculation for BG-normal form Hamiltonians $\mathcal{A}^{(\varepsilon)}$ and $\mathcal{R}^{(\varepsilon)}$ is performed on Maxima. In this subsection, we give the result of BG-normal form calculation performed on Maxima up to degree 6, which is described in terms of $J_{\ell}$.

Let $\mathcal{A}^{(\varepsilon)}(\xi, \eta), \mathcal{R}^{(\varepsilon)}(\xi, \eta)$ be BG normalized Hamiltonians up to degree 6 . If we set

$$
G=\varepsilon g, \quad F=\varepsilon f, \quad g^{2}+f^{2}=1,
$$

then the BG normalized Hamiltonians $\mathcal{A}^{(\varepsilon)}$ and $\mathcal{R}^{(\varepsilon)}$ can be put in the form

$$
\begin{aligned}
& \mathcal{A}^{(\varepsilon)}=\mathcal{A}+\varepsilon \mathcal{A}_{4}+\varepsilon^{2} \mathcal{A}_{6}, \\
& \mathcal{R}^{(\varepsilon)}=\mathcal{R}+\varepsilon \mathcal{R}_{4}+\varepsilon^{2} \mathcal{R}_{6},
\end{aligned}
$$


where $\mathcal{A}_{k}, \mathcal{R}_{k}, k=4,6$, are homogeneous polynomials of degree $k$ in $(\xi, \eta)$.

Since the canonical transformation $(x, y) \mapsto(\xi, \eta)$ is determined for a small value of the perturbation parameter $\varepsilon$, we may look on $\xi_{j}, \eta_{j}$ as

$$
x_{j} \fallingdotseq \xi_{j}, \quad y_{j} \fallingdotseq \eta_{j}, \quad j=1,2,3,4,
$$

so that

$$
A_{1}(x, y) \fallingdotseq \mathcal{A}(\xi, \eta), \quad R_{1}(x, y) \fallingdotseq \mathcal{R}(\xi, \eta) .
$$

In what follows, we give the explicit expression of the perturbation term in BG normal form. In the case of the perturbed harmonic oscillator, Maxima provides us with polynomials $\mathcal{A}_{k}, k=4,6$, which can be described in terms of $J_{j+1}, J_{j+4}$, $j=1,2,3$, as

$$
\begin{aligned}
\mathcal{A}_{1}= & 2 J_{1}, \\
\mathcal{A}_{4}= & 2 J_{1} J_{7} g+2 J_{0} J_{7} g+2 J_{1} J_{4} g-2 J_{0} J_{4} g+12 J_{1} J_{6} f+4 J_{0} J_{6} f-12 J_{1} J_{3} f \\
& +4 J_{0} J_{3} f \\
\mathcal{A}_{6}= & 3 J_{1} J_{7}^{2} g^{2}-3 J_{0} J_{7}^{2} g^{2}+6 J_{1} J_{6}^{2} g^{2}-4 J_{1} J_{3} J_{6} g^{2}+6 J_{1} J_{5}^{2} g^{2}-4 J_{1} J_{2} J_{5} g^{2} \\
& -3 J_{1} J_{4}^{2} g^{2}+3 J_{0} J_{4}^{2} g^{2}+12 J_{0} J_{1}^{2} g^{2}-12 J_{0}^{2} J_{1} g^{2}-24 J_{1} J_{6} J_{7} f g-16 J_{0} J_{6} J_{7} f g \\
& +32 J_{1} J_{3} J_{7} f g+8 J_{0} J_{3} J_{7} f g-32 J_{1} J_{4} J_{6} f g+8 J_{0} J_{4} J_{6} f g+24 J_{1} J_{3} J_{4} f g \\
& -16 J_{0} J_{3} J_{4} f g-136 J_{1} J_{7}^{2} f^{2}-220 J_{1} J_{6}^{2} f^{2}-36 J_{0} J_{6}^{2} f^{2}+240 J_{1} J_{3} J_{6} f^{2} \\
& -136 J_{1} J_{5}^{2} f^{2}-84 J_{1} J_{3}^{2} f^{2}+36 J_{0} J_{3}^{2} f^{2}-272 J_{0} J_{1}^{2} f^{2}+208 J_{0}^{2} J_{1} f^{2} .
\end{aligned}
$$

On setting $J_{0}=\frac{1}{2} \mu$ and $J_{1}=2 \kappa$, the perturbed Hamiltonian $\mathcal{A}^{(\varepsilon)}$ can be viewed as defined on the iso-energetic orbit space $M_{\mu, \kappa}^{-}=\left(\mathcal{A}^{-1}(4 \kappa) \cap \Phi^{-1}(\mu)\right) /(U(1) \times U(1))$.

In the case of the repulsive oscillator, the polynomials $\mathcal{R}_{k}, k=4,6$, prove to be written in terms of $\tilde{J}_{k+1}:=\left(J_{k+1}+J_{k+4}\right) / 2, J_{k+8}, j=1,2,3$, as follows:

$$
\begin{aligned}
\mathcal{R}_{1}= & -2 J_{8}, \\
\mathcal{R}_{4}= & -4 J_{0} J_{11} g+4 \tilde{J}_{4} J_{8} g-24 J_{8} J_{10} f-8 J_{0} \tilde{J}_{3} f, \\
\mathcal{R}_{6}= & -12 J_{8} J_{11}^{2} g^{2}-12 J_{0} \tilde{J}_{4} J_{11} g^{2}-2 J_{8} J_{10}^{2} g^{2}-2 J_{8} J_{9}^{2} g^{2}+12 J_{8}^{3} g^{2}+12 \tilde{J}_{4}^{2} J_{8} g^{2} \\
& +10 \tilde{J}_{3}^{2} J_{8} g^{2}+10 \tilde{J}_{2}^{2} J_{8} g^{2}+48 J_{0} J_{10} J_{11} f g+16 \tilde{J}_{3} J_{8} J_{11} f g-112 \tilde{J}_{4} J_{8} J_{10} f g \\
& -16 J_{0} \tilde{J}_{3} \tilde{J}_{4} f g-72 J_{8} J_{11}^{2} f^{2}+336 J_{8} J_{10}^{2} f^{2}+144 J_{0} \tilde{J}_{3} J_{10} f^{2}-72 J_{8} J_{9}^{2} f^{2} \\
& +208 J_{8}^{3} f^{2}+72 \tilde{J}_{4}^{2} J_{8} f^{2}+144 \tilde{J}_{3}^{2} J_{8} f^{2}+72 \tilde{J}_{2}^{2} J_{8} f^{2} .
\end{aligned}
$$

Setting $J_{0}=\frac{1}{2} \mu$ and $J_{8}=-2 \kappa$, we obtain the perturbed Hamiltonian $\mathcal{R}^{(\varepsilon)}$ defined on the iso-energetic orbit space $M_{\mu, \kappa}^{+}=\left(\mathcal{R}^{-1}(4 \kappa) \cap \Phi^{-1}(\mu)\right) /(U(1) \times \mathbb{R})$. 


\subsection{Perturbed MIC-Kepler problems in normal form}

We now show that the perturbed Hamiltonians $\mathcal{A}^{(\varepsilon)}$ and $\mathcal{R}^{(\varepsilon)}$ can be viewed as Hamiltonians on the reduced iso-energetic orbit space $M_{\mu, \kappa}^{\mp}$ for the perturbed MIC-Kepler problem at negative and positive energies, respectively. Let

$$
\mathcal{H}_{c}(\xi, \eta):=\left.H_{c}(x, y)\right|_{(x, y)=(\xi, \eta)}
$$

be the unperturbed Hamiltonian expressed in terms of $\xi$ and $\eta$. Then, it is related to $\mathcal{A}$ and $\mathcal{R}$ by

$$
4 \rho\left(\mathcal{H}_{c}+\frac{1}{8}\right)=\mathcal{A}-4 \kappa, \quad 4 \rho\left(\mathcal{H}_{c}-\frac{1}{8}\right)=\mathcal{R}-4 \kappa,
$$

respectively.

In the case of the harmonic oscillator, the Poisson bracket of $\mathcal{H}_{c}$ and each of the perturbation terms $G_{k}^{-}$is written out as

$$
\left\{\mathcal{H}_{c}, G_{k}^{-}\right\}=\left\{\frac{1}{4 \rho}(\mathcal{A}-4 \kappa), G_{k}^{-}\right\}=(\mathcal{A}-4 \kappa)\left\{\frac{1}{4 \rho}, G_{k}^{-}\right\} .
$$

This implies that $G_{k}^{-}, k=3,4, \cdots, r$, and $\mathcal{H}_{c}$ Poisson-commute on the energy manifold $\mathcal{H}_{c}^{-1}(-1 / 8)=\mathcal{A}^{-1}(4 \kappa)$. A similar statement is true in the case of the repulsive oscillator: $G_{k}^{+}, k=3,4, \cdots, r$, and $\mathcal{H}_{c}$ Poisson-commute on the energy manifold $\mathcal{H}_{c}^{-1}(1 / 8)=\mathcal{R}^{-1}(4 \kappa)$.

Since $\left\{\mathcal{H}_{c}, G_{k}^{\mp}\right\}=0, k=3,4, \cdots, r$, hold on respective energy manifolds $\mathcal{H}_{c}^{-1}(-1 / 8)=\mathcal{A}^{-1}(4 \kappa)$ and $\mathcal{H}_{c}^{-1}(1 / 8)=\mathcal{R}^{-1}(4 \kappa)$, the $G_{k}^{\mp}$ can be regarded as perturbation terms in normal form if restricted on $\mathcal{H}_{c}^{-1}(\mp 1 / 8)$, and further reduced by the $U(1) \times U(1)$ symmetry and by the $U(1) \times \mathbb{R}$ symmetry to functions on the respective iso-energetic orbit spaces $\mathcal{H}_{c}^{-1}(-1 / 8) \cap \Phi^{-1}(\mu) /(U(1) \times U(1)) \cong M_{\mu, \kappa}^{-}$ and $\left.\mathcal{H}_{c}^{-1}(1 / 8) \cap \Phi^{-1}(\mu) /(U(1) \times \mathbb{R})\right) \cong M_{\mu, \kappa}^{+}$.

A remaining task we have to do is to check whether the $\mathcal{A}^{(\varepsilon)}$ and $\mathcal{R}^{(\varepsilon)}$ give rise to perturbations of the MIC-Kepler problem in normal form at negative and positive energies, respectively. If the perturbation parameter $\varepsilon$ is small, the BG-normal form Hamiltonians $\mathcal{A}^{(\varepsilon)}, \mathcal{R}^{(\varepsilon)}$ given in (118a), (118b) are good approximations to $A^{(\varepsilon)}$ and $R^{(\varepsilon)}$, respectively, that is,

$$
\begin{aligned}
& A^{(\varepsilon)}=A_{1}+N_{A}^{(\varepsilon)} \fallingdotseq \mathcal{A}+\mathcal{N}_{A}^{(\varepsilon)}=\mathcal{A}^{(\varepsilon)} \\
& R^{(\varepsilon)}=R_{1}+N_{R}^{(\varepsilon)} \fallingdotseq \mathcal{R}+\mathcal{N}_{R}^{(\varepsilon)}=\mathcal{R}^{(\varepsilon)}
\end{aligned}
$$


According to the sign of the energy, we may define a perturbed Hamiltonian $\mathcal{H}_{c}^{(\varepsilon)}$ to be

$$
\mathcal{H}_{c}^{(\varepsilon)}:=\mathcal{H}_{c}+ \begin{cases}\frac{1}{4 \rho} \mathcal{N}_{A}^{(\varepsilon)} & \text { if } E=-\frac{1}{8} \\ \frac{1}{4 \rho} \mathcal{N}_{R}^{(\varepsilon)} & \text { if } E=\frac{1}{8} .\end{cases}
$$

The Hamiltonian $\mathcal{H}_{c}^{(\varepsilon)}$ thus defined should be a good approximation to the original perturbed Hamiltonian $H_{c}^{(\varepsilon)}=H_{c}+N_{c}^{(\varepsilon)}$ in the negative- and positive-energy cases.

A question arises here as to whether the $\mathcal{H}_{c}^{(\varepsilon)}$ given in (133) commute with the unperturbed term $\mathcal{H}_{c}$ or not; $\left\{\mathcal{H}_{c}, \mathcal{H}_{c}^{(\varepsilon)}\right\}=0$ or $\neq 0$. In obtaining $\mathcal{H}_{c}^{(\varepsilon)}$, we have assumed tacitly that the energy values $E=\mp \frac{1}{8}$ are fixed. We now ask if the energy value may be varied in such a manner that a perturbed Hamiltonian and the original Hamiltonian Poisson-commute. If perturbed, the energy of the system may change accordingly. Introducing a parameter $\tilde{\lambda}>0$, we put the energy change in the form

$$
E=\mp \frac{1}{8} \longrightarrow \tilde{E}=E+\Delta E=\mp \frac{\tilde{\lambda}^{2}}{8} .
$$

We note that if $\varepsilon=0$ then the increment of energy is zero, $\Delta E=0$, and $\tilde{\lambda}$ is unchanged, $\tilde{\lambda}=1$. In place of (114), the perturbed harmonic and repulsive oscillators may be defined to be

$$
A_{\tilde{\lambda}}^{(\varepsilon)}=A_{\tilde{\lambda}}+N_{A}^{(\varepsilon)}, \quad R_{\tilde{\lambda}}^{(\varepsilon)}=R_{\tilde{\lambda}}+N_{R}^{(\varepsilon)},
$$

respectively, and they are transformed, in the same manner as in (118a) and $(118 b)$, into

$$
\mathcal{A}_{\tilde{\lambda}}^{(\varepsilon)}=\mathcal{A}_{\tilde{\lambda}}+\mathcal{N}_{A}^{(\varepsilon)}, \quad \mathcal{R}_{\tilde{\lambda}}^{(\varepsilon)}=\mathcal{R}_{\tilde{\lambda}}+\mathcal{N}_{R}^{(\varepsilon)},
$$

respectively, where $\mathcal{A}_{\tilde{\lambda}}^{(\varepsilon)}$ and $\mathcal{R}_{\tilde{\lambda}}^{(\varepsilon)}$ are put in the BG-normal form, $\left\{\mathcal{A}_{\tilde{\lambda}}, \mathcal{A}_{\tilde{\lambda}}^{(\varepsilon)}\right\}=0$ and $\left\{\mathcal{R}_{\tilde{\lambda}}, \mathcal{R}_{\tilde{\lambda}}^{(\varepsilon)}\right\}=0$, respectively.

We compare two energy manifolds given, respectively, by

$$
\mathcal{A}^{-1}(4 \kappa)=\mathcal{H}_{c}^{-1}\left(-\frac{1}{8}\right), \quad\left(\mathcal{A}_{\tilde{\lambda}}^{(\varepsilon)}\right)^{-1}(4 \kappa)=\left(\mathcal{H}_{c}^{(\varepsilon)}\right)^{-1}\left(-\frac{\tilde{\lambda}^{2}}{8}\right)
$$

and those given, respectively, by

$$
\mathcal{R}^{-1}(4 \kappa)=\mathcal{H}_{c}^{-1}\left(\frac{1}{8}\right), \quad\left(\mathcal{R}_{\tilde{\lambda}}^{(\varepsilon)}\right)^{-1}(4 \kappa)=\left(\mathcal{H}_{c}^{(\varepsilon)}\right)^{-1}\left(\frac{\tilde{\lambda}^{2}}{8}\right)
$$

The energy manifolds given in (137) (resp. in (138)) are different from each other, but when $\varepsilon=0$, they coincide with each other. Since the deformation is smooth 
in $\tilde{\lambda}$ and $\varepsilon$ for $\varepsilon$ small enough, the difference between the energy manifolds given in (137) (resp. in (138)) should be small, and there should exist diffeomorphisms

$$
\begin{aligned}
& \psi_{\tilde{\lambda}}^{-}: \mathcal{A}^{-1}(4 \kappa) \rightarrow\left(\mathcal{A}_{\tilde{\lambda}}^{(\varepsilon)}\right)^{-1}(4 \kappa), \\
& \psi_{\tilde{\lambda}}^{+}: \mathcal{R}^{-1}(4 \kappa) \rightarrow\left(\mathcal{R}_{\tilde{\lambda}}^{(\varepsilon)}\right)^{-1}(4 \kappa),
\end{aligned}
$$

which satisfy

$$
\begin{array}{ll}
\left(\psi_{\tilde{\lambda}}^{-}\right)_{*}\left(X_{\mathcal{A}}\right)_{p}=\left(X_{\mathcal{A}_{\tilde{\lambda}}}\right)_{\psi_{\tilde{\lambda}}^{-}(p)}, & p \in \mathcal{A}^{-1}(4 \kappa), \\
\left(\psi_{\tilde{\lambda}}^{+}\right)_{*}\left(X_{\mathcal{R}}\right)_{p}=\left(X_{\mathcal{R}_{\tilde{\lambda}}}\right)_{\psi_{\tilde{\lambda}}^{+}(p)}, & p \in \mathcal{R}^{-1}(4 \kappa),
\end{array}
$$

respectively. Note that if $\varepsilon=0$ then $\psi_{\tilde{\lambda}}^{-}\left(\operatorname{resp} . \psi_{\tilde{\lambda}}^{+}\right)$becomes the identity map on $\mathcal{A}^{-1}(4 \kappa)\left(\operatorname{resp} . \mathcal{R}^{-1}(4 \kappa)\right)$, and if $\varepsilon$ is small enough, then $\psi_{\tilde{\lambda}}^{-}\left(\operatorname{resp} . \psi_{\tilde{\lambda}}^{+}\right)$is close to the identity map.

Let $\psi^{\mp}$ be extensions of (139a) and (139b) defined in the neighborhood of $\mathcal{A}^{-1}(4 \kappa)$ and of $\mathcal{R}^{-1}(4 \kappa)$, respectively;

$$
\begin{array}{ll}
\left.\psi^{-}\right|_{\mathcal{A}^{-1}(4 \kappa)}(p)=\psi_{\tilde{\lambda}}^{-}(p), & p \in \mathcal{A}^{-1}(4 \kappa), \\
\left.\psi^{+}\right|_{\mathcal{R}^{-1}(4 \kappa)}(p)=\psi_{\tilde{\lambda}}^{+}(p), & p \in \mathcal{R}^{-1}(4 \kappa) .
\end{array}
$$

We define a perturbed Hamiltonian $\tilde{\mathcal{H}}_{c}^{(\varepsilon)}$, according to whether the energy is negative or positive, to be

$$
\tilde{\mathcal{H}}_{c}^{(\varepsilon)}:=\mathcal{H}_{c}^{(\varepsilon)} \circ \psi^{\mp},
$$

where $\mathcal{H}_{c}^{(\varepsilon)}$ is defined by (133). In the negative-energy case, for an arbitrary $p \in \mathcal{A}^{-1}(4 \kappa)$, the Poisson bracket of $\mathcal{H}_{c}$ and $\tilde{\mathcal{H}}_{c}^{(\varepsilon)}$ is calculated as follows:

$$
\begin{aligned}
\left\{\mathcal{H}_{c}, \tilde{\mathcal{H}}_{c}{ }^{(\varepsilon)}\right\}(p) & =\left\{\frac{1}{4 \rho}(\mathcal{A}-4 \kappa), \tilde{\mathcal{H}}_{c}^{(\varepsilon)}\right\}(p) \\
& =-\frac{1}{4 \rho} X_{\mathcal{A}} \tilde{\mathcal{H}}_{c}^{(\varepsilon)}(p)=-\frac{1}{4 \rho} X_{\mathcal{A}}\left(\psi^{-}\right)^{*} \mathcal{H}_{c}^{(\varepsilon)}(p) \\
& =-\frac{1}{4 \rho}\left(\psi^{-}\right)_{*}\left(X_{\mathcal{A}}\right)_{p} \mathcal{H}_{c}^{(\varepsilon)}=-\frac{1}{4 \rho}\left(X_{\left.\mathcal{A}_{\tilde{\lambda}}\right)_{\psi_{\tilde{\lambda}}^{-}(p)} \mathcal{H}_{c}^{(\varepsilon)}}\right. \\
& =\frac{1}{4 \rho}\left\{\mathcal{A}_{\tilde{\lambda}}, \mathcal{H}_{c}^{(\varepsilon)}\right\}\left(\psi_{\tilde{\lambda}}^{-}(p)\right) \\
& =\frac{1}{4 \rho}\left\{\mathcal{A}_{\tilde{\lambda}}, \frac{1}{4 \rho}\left(\mathcal{A}^{(\varepsilon)}-4 \kappa\right)\right\}\left(\psi_{\tilde{\lambda}}^{-}(p)\right) \\
& =\frac{1}{16 \rho^{2}}\left\{\mathcal{A}_{\tilde{\lambda}}, \mathcal{A}^{(\varepsilon)}-4 \kappa\right\}\left(\psi_{\tilde{\lambda}}^{-}(p)\right) .
\end{aligned}
$$


In the positive-energy case, we can show in the same manner as above that

$$
\left\{\mathcal{H}_{c}, \tilde{\mathcal{H}}_{c}^{(\varepsilon)}\right\}(p)=\frac{1}{16 \rho^{2}}\left\{\mathcal{R}_{\tilde{\lambda}}, \mathcal{R}^{(\varepsilon)}-4 \kappa\right\}\left(\psi_{\tilde{\lambda}}^{+}(p)\right), \quad p \in \mathcal{R}^{-1}(4 \kappa) .
$$

The above calculation implies that if $\tilde{\lambda}=1$ the right-hand sides of (143) and (144) vanish, so that $\left\{\mathcal{H}_{c}, \tilde{\mathcal{H}}_{c}^{(\varepsilon)}\right\}=0$ on $\mathcal{A}^{-1}(4 \kappa)$ and on $\mathcal{R}^{-1}(4 \kappa)$. Since $\tilde{\mathcal{H}}_{c}^{(\varepsilon)}$ becomes $\mathcal{H}_{c}^{(\varepsilon)}$ for $\tilde{\lambda}=1$, we see that $\left\{\mathcal{H}_{c}, \mathcal{H}_{c}^{(\varepsilon)}\right\}=0$ on $\mathcal{A}^{-1}(4 \kappa)$ and on $\mathcal{R}^{-1}(4 \kappa)$. If projected on the iso-energetic orbit spaces $M_{\mu, \kappa}^{\mp}$, this relation is brought into $\left\{\mathcal{H}_{\mu}, \mathcal{H}_{\mu}^{(\varepsilon)}\right\}=0$, where $\mathcal{H}_{\mu}$ and $\mathcal{H}_{\mu}^{(\varepsilon)}$ are projections of (131) and (133), respectively. Our question is now solved to give the following theorem:

Theorem 6.1 The perturbed harmonic and repulsive oscillator Hamiltonians $\mathcal{A}^{(\varepsilon)}$ and $\mathcal{R}^{(\varepsilon)}$ in BG-normal form determine a perturbed Hamiltonian $\mathcal{H}_{\mu}^{(\varepsilon)}$ for the perturbed MIC-Kepler problem, which is in normal form if projected on the iso-energetic orbit spaces $M_{\mu, \kappa}^{\mp}$, where $\mathcal{H}_{\mu}^{(\varepsilon)}$ are the reduced Hamiltonian from (133) onto $T^{*} \dot{\mathbb{R}}^{3}$.

We here remark that this theorem is independent of the choice of perturbations.

\section{Poisson mechanics for perturbed systems}

In this section, we apply the Poisson mechanics to the perturbed Hamiltonians obtained in Sec. 6.

\subsection{Hamiltonian flows generated by leading terms}

Since the perturbed Hamiltonians $\mathcal{A}^{(\varepsilon)}$ and $\mathcal{R}^{(\varepsilon)}$ are put in the form (122a) and (122b), respectively, we are interested in the first perturbation term $\mathcal{A}_{4}$ and $\mathcal{R}_{4}$, which are the most influential terms in the perturbed Hamiltonians.

In terms of $\boldsymbol{L}^{\mp}, \boldsymbol{K}^{\mp}$, the leading terms (126) and (129) can be rewritten as

$$
\begin{aligned}
& \mathcal{A}_{4}=-J_{1}\left(4 L_{3}^{-} g+24 K_{2}^{-} f\right)-J_{0}\left(4 K_{3}^{-} g+8 L_{2}^{-} f\right), \\
& \mathcal{R}_{4}=-J_{8}\left(4 L_{3}^{+} g-24 K_{2}^{+} f\right)+J_{0}\left(4 K_{3}^{+} g+8 L_{2}^{+} f\right),
\end{aligned}
$$

respectively. Setting $J_{1}=2 \kappa, J_{8}=-2 \kappa$, and $J_{0}=\mu / 2$, and introducing $\alpha, \beta, \tilde{\mu}$, in place of the parameters (121), by

$$
\alpha=-8 g \kappa, \quad \beta=-48 f \kappa, \quad \alpha^{2}+\beta^{2}=1, \quad \tilde{\mu}=\frac{\mu}{12 \kappa},
$$


we put Eqs.(145a) and (145b) in the form

$$
\begin{aligned}
& \mathcal{A}_{4}=L_{3}^{-} \alpha+K_{2}^{-} \beta+\tilde{\mu}\left(3 K_{3}^{-} \alpha+L_{2}^{-} \beta\right), \\
& \mathcal{R}_{4}=-L_{3}^{+} \alpha+K_{2}^{+} \beta-\tilde{\mu}\left(3 K_{3}^{+} \alpha+L_{2}^{+} \beta\right),
\end{aligned}
$$

respectively, which are approximate Hamiltonians on $\tilde{M}_{\mu, \kappa}^{\mp}$. If $\tilde{\mu}=0$ in particular, then the $\mathcal{A}_{4}$ becomes $\mathcal{A}_{4}=L_{3}^{-} \alpha+K_{2}^{-} \beta$, which coincides with the result given in [6]. The equations of motion (107) for the Hamiltonian $\mathcal{A}_{4}$ is now expressed as

$$
\frac{d}{d t}\left(\begin{array}{c}
\boldsymbol{L}^{-} \\
\boldsymbol{K}^{-}
\end{array}\right)=Q_{1}\left(\begin{array}{c}
\boldsymbol{L}^{-} \\
\boldsymbol{K}^{-}
\end{array}\right) \text {with } Q_{1}=\left(\begin{array}{cccccc}
0 & -\alpha & \beta \tilde{\mu} & 0 & -3 \alpha \tilde{\mu} & \beta \\
\alpha & 0 & 0 & 3 \alpha \tilde{\mu} & 0 & 0 \\
-\beta \tilde{\mu} & 0 & 0 & -\beta & 0 & 0 \\
0 & -3 \alpha \tilde{\mu} & \beta & 0 & -\alpha & \beta \tilde{\mu} \\
3 \alpha \tilde{\mu} & 0 & 0 & \alpha & 0 & 0 \\
-\beta & 0 & 0 & -\beta \tilde{\mu} & 0 & 0
\end{array}\right) .
$$

In comparison with this, Eq. (107) for the Hamiltonian $\mathcal{R}_{4}$ is written as

$$
\frac{d}{d t}\left(\begin{array}{c}
\boldsymbol{L}^{+} \\
\boldsymbol{K}^{+}
\end{array}\right)=Q_{2}\left(\begin{array}{c}
\boldsymbol{L}^{+} \\
\boldsymbol{K}^{+}
\end{array}\right) \text {with } Q_{2}=\left(\begin{array}{cccccc}
0 & \alpha & -\beta \tilde{\mu} & 0 & 3 \alpha \tilde{\mu} & \beta \\
-\alpha & 0 & 0 & -3 \alpha \tilde{\mu} & 0 & 0 \\
\beta \tilde{\mu} & 0 & 0 & -\beta & 0 & 0 \\
0 & -3 \alpha \tilde{\mu} & -\beta & 0 & \alpha & -\beta \tilde{\mu} \\
3 \alpha \tilde{\mu} & 0 & 0 & -\alpha & 0 & 0 \\
\beta & 0 & 0 & \beta \tilde{\mu} & 0 & 0
\end{array}\right) .
$$

The properties of the dynamical systems (148) and (149) are determined by the respective four non-zero eigenvalues of $Q_{1}$ and $Q_{2}$, respectively. Since each of $\tilde{M}_{\mu, \kappa}^{\mp}$ is symplectic leaf in $\mathbb{R}^{6}$ and invariant under the flow of (107), the eigenvalues of both $Q_{1}$ and $Q_{2}$ should contain two zero eigenvalues.

The eigenvalues of $Q_{1}$ other than zero are given by

$$
\begin{aligned}
& \pm i \sqrt{\beta^{2} \tilde{\mu}^{2}+9 \alpha^{2} \tilde{\mu}^{2}-2 \beta^{2} \tilde{\mu}-6 \alpha^{2} \tilde{\mu}+\beta^{2}+\alpha^{2}}=: \pm i \lambda_{1}, \\
& \pm i \sqrt{\beta^{2} \tilde{\mu}^{2}+9 \alpha^{2} \tilde{\mu}^{2}+2 \beta^{2} \tilde{\mu}+6 \alpha^{2} \tilde{\mu}+\beta^{2}+\alpha^{2}}=: \pm i \lambda_{2}
\end{aligned}
$$

which are pure imaginary numbers for all $\tilde{\mu} \in \mathbb{R},|\alpha| \leq 1,|\beta| \leq 1$, where $\alpha$ and $\beta$ are subject to the condition (146). It then turns out that the periodic orbits of the MIC-Kepler problem at a negative energy are linearly stable in the presence of week electric and magnetic fields. If $\tilde{\mu}=0$, then $\lambda_{1}$ and $\lambda_{2}$ coincide. This fact 
is known for a perturbed Kepler problem [3] with $f_{e}=0$. If $\alpha=0$ and $\tilde{\mu}=1$, then $\lambda_{1}=0$, and if $\alpha=0$ and $\tilde{\mu}=-1$, then $\lambda_{2}=0$.

The eigenvalues of $Q_{2}$ other than zero are

$$
\begin{aligned}
& \pm \sqrt{-\beta^{2} \tilde{\mu}^{2}+9 \alpha^{2} \tilde{\mu}^{2}+2 i \beta^{2} \tilde{\mu}-6 i \alpha^{2} \tilde{\mu}+\beta^{2}-\alpha^{2}} \\
& \pm \sqrt{-\beta^{2} \tilde{\mu}^{2}+9 \alpha^{2} \tilde{\mu}^{2}-2 i \beta^{2} \tilde{\mu}+6 i \alpha^{2} \tilde{\mu}+\beta^{2}-\alpha^{2}}
\end{aligned}
$$

It then turns out that if $\tilde{\mu}=0$ and if $\alpha^{2}>\beta^{2}$ the non-zero eigenvalues become pure imaginary numbers, which implies that a scattering orbit can be linearly stable in the presence of suitable electric and magnetic fields.

For the perturbed harmonic oscillator case, after an appropriate coordinate transformation, the equations of motion (148) turns into

$$
\frac{d W^{-}}{d t}=\left(\begin{array}{cccccc}
0 & -\lambda_{1} & 0 & 0 & 0 & 0 \\
\lambda_{1} & 0 & 0 & 0 & 0 & 0 \\
0 & 0 & 0 & -\lambda_{2} & 0 & 0 \\
0 & 0 & \lambda_{2} & 0 & 0 & 0 \\
0 & 0 & 0 & 0 & 0 & 0 \\
0 & 0 & 0 & 0 & 0 & 0
\end{array}\right) W^{-}
$$

where $W^{-}=\left(W_{1}^{-}, \cdots, W_{6}^{-}\right)^{T}$ are local coordinates on $\mathbb{R}^{6}$ defined through

$$
T^{-} W^{-}=\left(\begin{array}{l}
\boldsymbol{L}^{-} \\
\boldsymbol{K}^{-}
\end{array}\right) \text {with } T^{-}=\left(\begin{array}{ccccccc}
\lambda_{1} & 0 & \lambda_{2} & 0 & 0 & 0 \\
0 & \alpha-3 \alpha \tilde{\mu} & 0 & 3 \alpha \tilde{\mu}+\alpha & 3 \beta \mu^{2}-\beta & 0 \\
0 & \beta-\beta \tilde{\mu} & 0 & -\beta \mu-\beta & 0 & 3 \alpha \tilde{\mu}^{2}-\alpha \\
-\lambda_{1} & 0 & \lambda_{2} & 0 & 0 & 0 \\
0 & 3 \alpha \tilde{\mu}-\alpha & 0 & 3 \alpha \tilde{\mu}+\alpha & 2 \beta \tilde{\mu} & \beta \tilde{\mu}^{2}-\beta \\
0 & \beta \tilde{\mu}-\beta & 0 & -\beta \tilde{\mu}-\beta & 9 \alpha \tilde{\mu}^{2}-\alpha & -2 \alpha \tilde{\mu}
\end{array}\right) .
$$

Equation (152) implies that the conditions $W_{1}^{-}=\cdots=W_{4}^{-}=0, W_{4}^{-}=c_{4}, W_{5}^{-}=$ $c_{5}$ with $c_{4}, c_{5}$ constants determine zeros of the Hamiltonian vector field associated with $\mathcal{A}^{(\varepsilon)}=\mathcal{A}+\varepsilon \mathcal{A}_{4}$ on $\tilde{M}_{\mu, \kappa}^{-}$or $M_{\mu, \kappa}^{-}$. Since these zeros are fixed points against perturbation, they are pulled back through the projection $H_{\mu}^{-1}\left(-\frac{1}{8}\right) \rightarrow M_{\mu, \kappa}^{-}$to determine periodic orbits of the perturbed MIC-Kepler problem associated with $\mathcal{A}^{(\varepsilon)}=\mathcal{A}+\varepsilon \mathcal{A}_{4}$. The number of the periodic orbits is equal to the Euler index of $S^{2} \times S^{2}$.

For the perturbed repulsive oscillator case, if $\tilde{\mu}=0$ and $\alpha^{2}>\beta^{2}$, then after an 
appropriate coordinate transformation the equations of motion (149) turns into

$$
\frac{d W^{+}}{d t}=\left(\begin{array}{cccccc}
0 & -c & 0 & 0 & 0 & 0 \\
c & 0 & 0 & 0 & 0 & 0 \\
0 & 0 & 0 & -c & 0 & 0 \\
0 & 0 & c & 0 & 0 & 0 \\
0 & 0 & 0 & 0 & 0 & 0 \\
0 & 0 & 0 & 0 & 0 & 0
\end{array}\right) W^{+}
$$

where $c=\sqrt{\alpha^{2}-\beta^{2}}$, and where $W^{+}=\left(W_{1}^{+}, \cdots, W_{6}^{+}\right)^{T}$ are local coordinates on $\mathbb{R}^{6}$ defined through

$$
T^{+} W^{+}=\left(\begin{array}{l}
\boldsymbol{L}^{+} \\
\boldsymbol{K}^{+}
\end{array}\right) \text {with } T^{+}=\left(\begin{array}{cccccc}
c & 0 & 0 & 0 & 0 & 0 \\
0 & -\alpha & 0 & 0 & \beta & 0 \\
0 & 0 & \beta & 0 & 0 & \alpha \\
0 & 0 & 0 & c & 0 & 0 \\
0 & 0 & \alpha & 0 & 0 & \beta \\
0 & \beta & 0 & 0 & -\alpha & 0
\end{array}\right) .
$$

Let $\sigma$ be a parameter and let $R_{\sigma}(t)$ be an $S O(2)$ action defined by

$$
R_{\sigma}(t):=\left(\begin{array}{cc}
\cos (\sigma t) & -\sin (\sigma t) \\
\sin (\sigma t) & \cos (\sigma t)
\end{array}\right), \quad t \in \mathbb{R} .
$$

Then, the Hamiltonian flow $\phi_{\mathcal{A}_{4}}^{t}: \mathbb{R}^{6} \rightarrow \mathbb{R}^{6}: W^{-} \mapsto \phi_{\mathcal{A}_{4}}^{t}\left(W^{-}\right)$generated by $\mathcal{A}_{4}$ can be expressed as

$$
\phi_{\mathcal{A}_{4}}^{t}:\left(\begin{array}{ccc}
R_{\lambda_{1}}(t) & 0 & 0 \\
0 & R_{\lambda_{2}}(t) & 0 \\
0 & 0 & I_{2}
\end{array}\right) .
$$

Similarly, when $\alpha^{2}>\beta^{2}$ and $\tilde{\mu}=0$, the Hamiltonian flow $\phi_{\mathcal{R}_{4} \mid \tilde{\mu}=0}^{t}: \mathbb{R}^{6} \rightarrow \mathbb{R}^{6}$ : $W^{+} \mapsto \phi_{\mathcal{R}_{4} \mid \tilde{\mu}=0}^{t}\left(W^{+}\right)$generated by $\left.\mathcal{R}_{4}\right|_{\tilde{\mu}=0}$ can be expressed as

$$
\phi_{\mathcal{R}_{4} \mid \tilde{\mu}=0}^{t}:\left(\begin{array}{ccc}
R_{c}(t) & 0 & 0 \\
0 & R_{c}(t) & 0 \\
0 & 0 & I_{2}
\end{array}\right) .
$$

\subsection{Second normal form}

If the Hamiltonian flows generated by the leading terms $\mathcal{A}_{4}$ and $\mathcal{R}_{4}$ are periodic, higher order terms can be averaged with respect to these flows. 
If the ratio $\lambda_{1} / \lambda_{2}$ is a rational number, i.e., $\frac{\lambda_{1}}{\lambda_{2}}=\frac{n_{1}}{n_{2}}$, where $n_{1}$ and $n_{2}$ are coprime positive integers, in the perturbed harmonic oscillator case, then the flow $\phi_{\mathcal{A}_{4}}^{t}$ becomes periodic with the period $T=T_{1} n_{1}=T_{2} n_{2}$ with $T_{i}=\frac{2 \pi}{\lambda_{i}}, i=1,2$. In the perturbed repulsive oscillator case with $\tilde{\mu}=0$ and $\alpha^{2}>\beta^{2}$, the Hamiltonian flow $\phi_{\mathcal{R}_{4} \mid \tilde{\mu}=0}^{t}$ is periodic with period $2 \pi / c$. In these cases, the higher-order terms $\mathcal{A}_{6}$ and $\mathcal{R}_{6}$ can be averaged with respect to these flows: The term $\mathcal{A}_{6}$ and $\mathcal{R}_{6}$ can be averaged by

$$
\tilde{\mathcal{A}}_{6}\left(W^{-}\right):=\frac{1}{T} \int_{0}^{T} \mathcal{A}_{6}\left(\phi_{\mathcal{A}_{4}}^{t}\left(W^{-}\right)\right) d t
$$

and by

$$
\tilde{\mathcal{R}}_{6}\left(W^{+}\right):=\left.\frac{c}{2 \pi} \int_{0}^{2 \pi / c} \mathcal{R}_{6}\right|_{\tilde{\mu}=0}\left(\phi_{\mathcal{R}_{4} \mid \tilde{\mu}=0}^{t}\left(W^{+}\right)\right) d t
$$

respectively.

The first perturbation term $\mathcal{A}_{4}$ and the averaged Hamiltonians $\tilde{\mathcal{A}}_{6}$ written in terms of $W^{-}$is shown to be given as follows:

$$
\begin{aligned}
\mathcal{A}_{4}= & W_{5}\left(\left(24 \alpha^{2}+3\right) \tilde{\mu}^{3}+\left(1-4 \alpha^{2}\right) \tilde{\mu}\right)+W_{6}\left(\left(1-4 \alpha^{2}\right) \tilde{\mu}^{2}-1\right), \\
\tilde{\mathcal{A}}_{6}= & \frac{W_{5}^{2} P_{6,1}}{36 \kappa}+\frac{W_{6}^{2} P_{6,2}}{36 \kappa}+\frac{\left(W_{2}^{2}+W_{1}^{2}\right) P_{6,3}}{12 \kappa}+\frac{\left(W_{4}^{2}+W_{3}^{2}\right) P_{6,4}}{36 \kappa}+\frac{W_{5} W_{6} P_{6,5}}{72 \kappa} \\
& -20 \alpha^{2} \kappa \tilde{\mu}^{2}+\frac{13 \kappa \tilde{\mu}^{2}}{2}+\frac{22 \alpha^{2} \kappa \tilde{\mu}}{3}-\frac{17 \kappa \tilde{\mu}}{6}
\end{aligned}
$$

where we have used $W_{j}$ for $W_{j}^{-}$and put $\beta^{2}:=1-\alpha^{2}$, and where $P_{i, j}$ are constants:

$$
\begin{aligned}
P_{6,1}= & \left(630 \alpha^{4}-531 \alpha^{2}-99\right) \tilde{\mu}^{4}+\left(-132 \alpha^{4}+183 \alpha^{2}-51\right) \tilde{\mu}^{3} \\
& +\left(-155 \alpha^{4}+184 \alpha^{2}-29\right) \tilde{\mu}^{2}+\left(44 \alpha^{4}-61 \alpha^{2}+17\right) \tilde{\mu}-3 \alpha^{4}+5 \alpha^{2}-2, \\
P_{6,2}= & \left(262 \alpha^{4}-2 \alpha^{2}-17\right) \tilde{\mu}^{4}+\left(51 \alpha^{2}-132 \alpha^{4}\right) \tilde{\mu}^{3}+\left(-63 \alpha^{4}-52 \alpha^{2}+34\right) \tilde{\mu}^{2} \\
& +\left(44 \alpha^{4}-17 \alpha^{2}\right) \tilde{\mu}-3 \alpha^{4}+20 \alpha^{2}-17, \\
P_{6,3}= & -\left(9 \alpha^{4}-9 \alpha^{2}\right) \tilde{\mu}^{3}-\left(12 \alpha^{2}-12 \alpha^{4}\right) \tilde{\mu}^{2}-\left(6 \alpha^{4}-6 \alpha^{2}\right) \tilde{\mu}+\alpha^{4}-\alpha^{2}, \\
P_{6,4}= & \left(27 \alpha^{4}-27 \alpha^{2}\right) \tilde{\mu}^{3}+\left(388 \alpha^{4}-128 \alpha^{2}-17\right) \tilde{\mu}^{2}+\left(194 \alpha^{4}+2 \alpha^{2}-34\right) \tilde{\mu} \\
& +3 \alpha^{4}+41 \alpha^{2}-17, \\
P_{6,5}= & -\left(1620 \alpha^{4}+486 \alpha^{2}+81\right) \tilde{\mu}^{5}-\left(-1056 \alpha^{4}+276 \alpha^{2}+51\right) \tilde{\mu}^{4} \\
& -\left(28 \alpha^{4}-1028 \alpha^{2}+28\right) \tilde{\mu}^{3}-\left(352 \alpha^{4}+40 \alpha^{2}-68\right) \tilde{\mu}^{2} \\
& -\left(-128 \alpha^{4}+318 \alpha^{2}-109\right) \tilde{\mu}+44 \alpha^{2}-17,
\end{aligned}
$$


The first perturbation term $\left.\mathcal{R}_{4}\right|_{\tilde{\mu}=0}$ and the averaged Hamiltonian $\tilde{\mathcal{R}}_{6}$ are shown to be expressed in terms of $W^{+}$as

$$
\begin{aligned}
\left.\mathcal{R}_{4}\right|_{\tilde{\mu}=0}= & W_{6}\left(1-2 \alpha^{2}\right) \\
\tilde{\mathcal{R}}_{6}= & -\frac{W_{1}^{2}+W_{2}^{2}}{48 \kappa}\left(26 \alpha^{4}-8 \alpha^{2}-3\right)+\frac{W_{3}^{2}+W_{4}^{2}}{48 \kappa}\left(2 \alpha^{4}+4 \alpha^{2}-3\right) \\
& +\frac{W_{5}^{2}}{24 \kappa}\left(10 \alpha^{4}+2 \alpha^{2}-3\right)-\frac{W_{6}^{2}}{24 \kappa}\left(30 \alpha^{4}-28 \alpha^{2}+7\right)-\frac{7 \alpha^{2} \kappa}{9}-\frac{13 \kappa}{18},
\end{aligned}
$$

respectively, where we have used $W_{j}$ for $W_{j}^{+}$and put $\beta^{2}:=1-\alpha^{2}$. Note that the condition $\alpha^{2}>\beta^{2}$ is equivalent to $\alpha>\frac{1}{\sqrt{2}}$.

As is expected, the averaged functions $\tilde{\mathcal{A}}_{6}$ and $\tilde{\mathcal{R}}_{6}$ are described in terms of invariants with respect to $\phi_{\mathcal{A}_{4}}^{t}$ and $\phi_{\mathcal{R}_{4} \mid \tilde{\mu}=0}^{t}$, respectively.

\section{Concluding remarks}

We have shown that the perturbed MIC-Kepler problem in BG normal form is described in the formulation of Poisson mechanics on the symmetry Lie algebra $\mathfrak{s u}(2) \oplus \mathfrak{s u}(2)$ or $\mathfrak{s l}(2, \mathbb{C})$, according as the energy is negative or positive. Though we have given perturbation terms of order less than seven, we can perform the normal form calculation on Maxima to get higher orders terms. However, to give those results by Maxima is not our purpose at present. What we want to emphasize is that the perturbation in normal form can be performed on an equal footing for the cases of both positive and negative energies. In fact, we have obtained the equation of motion Eq. (107) on respective iso-energetic orbit spaces (or on respective co-adjoint orbits of symmetry groups) in the similar form and performed the BG-normal form calculation in parallel for both positive and negative energies (see Eq. (120)).

From the viewpoint of symmetry, the MIC-Kepler problem and the Kepler problem seems to make no difference, since they have the same symmetry group. However, they expose difference if perturbed systems are taken into account. This is because perturbed systems are defined on co-adjoint orbits of the symmetry groups, and because those orbits are assigned by two parameters. We here summarize the difference between them. For a negative energy, the iso-energetic orbit spaces of the MIC-Kepler problem and of the Kepler problem are topologically the same, $S^{2} \times S^{2}$, but their geometric realizations are different from each other, as is seen already in Sec. 5.3. In fact, the geometric realization of the co-adjoint orbit of the symmetry group $S U(2) \times S U(2)$ (or equivalently the iso-energetic 
orbit space $M_{\mu, \kappa}^{-}$) for the MIC-Kepler problem at a negative energy is given by $S^{2}(|\mu+4 \kappa|) \times S^{2}(|\mu-4 \kappa|)$. For the Kepler problem with $\mu=0$, the radii of the spheres are the same but they are different from each other for the MIC-Kepler problem. In the cases of $\mu= \pm 4 \kappa$, one of the factor spaces becomes a singleton. In comparison with this, for the MIC-Kepler problem of positive energy, the geometric realization of the co-adjoint orbit of the symmetry group $S L(2, \mathbb{C})$ is the complex two-sphere $\left(\boldsymbol{q}_{1}+i \boldsymbol{q}_{2}\right)^{2}=(\mu-4 i \kappa)^{2}$, which is diffeomorphic with the tangent bundle over the real two-sphere. If $\mu= \pm 4 \kappa$, then the squared complex radius $(\mu-4 i \kappa)^{2}$ becomes pure imaginary. For the Kepler problem with $\mu=0$, it is real.

Further, the difference between the perturbed MIC-Kepler and Kepler problems are observed in the behavior of Hamiltonian flows on the co-adjoint orbits. We see in (147) that additional terms, the terms having the coefficient $\tilde{\mu}$, come into the perturbation terms $\mathcal{A}_{4}$ and $\mathcal{R}_{4}$. This gives rise to difference in the nonzero eigenvalues, $\lambda_{1}, \lambda_{2}$, of the matrix $Q_{1}$, as is seen in (150). In the case of the perturbed Kepler problem, one has $\lambda_{1}=\lambda_{2}$, but $\lambda_{1} \neq \lambda_{2}$ for the perturbed MIC-Kepler problem. This implies that the leading term generates a periodic Hamiltonian flow for the perturbed Kepler problem, but the corresponding flow for the perturbed MIC-Kepler problem is not periodic in general, except for the case where $\lambda_{1} / \lambda_{2}$ is a rational number.

Though the monodromy is studied in [3-11] for the perturbed Kepler problem, this article has not treated the monodromy for the perturbed MIC-Kepler problem, which will be a future problem.

In conclusion, we make a remark on the mapping $\boldsymbol{z} \mapsto \sum_{j} z_{j} \sigma_{j}$ defined in (86). This map is well explained in [24], and of course used in a group representation theory by the orbit method (see [25], for example). Further, since $S L(2, \mathbb{C}) / \mathbb{Z}_{2} \cong$ $S O(3, \mathbb{C})$ and since mechanics of the top is described on a (co)adjoint orbit $S^{2}$ of $S O(3)$, the co-adjoint orbit of $S L(2, \mathbb{C})$ can carry mechanics for an extension of the top. This system is studied in the name of the $S L(2, \mathbb{C})$ Euler-Arnold top (see [26], for example).

A list of frequently used symbols. 


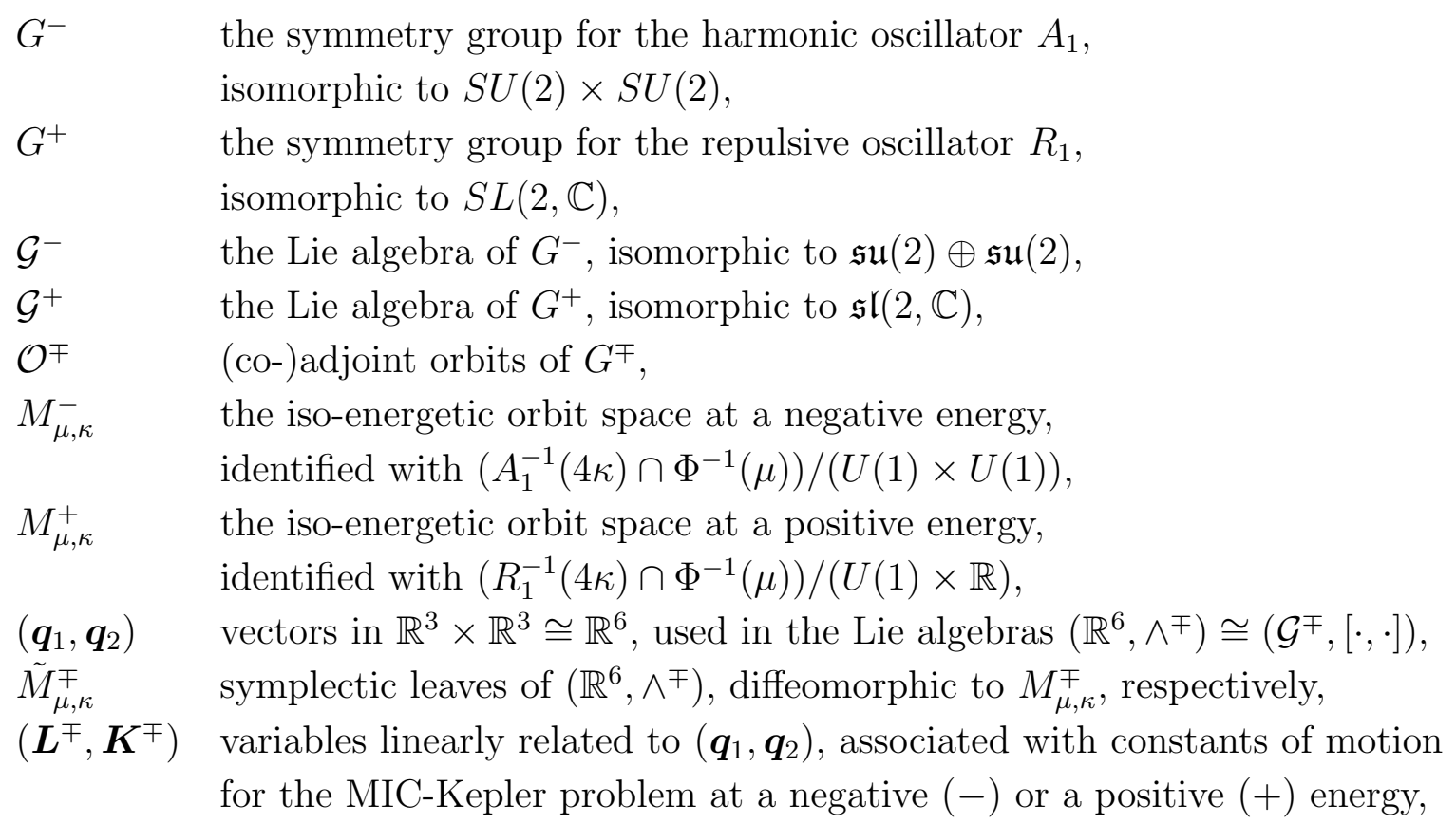

\section{Acknowledgment}

This work is partially supported by Grant-in-Aid for Scientific Research, JSPS, Scientific Research (C) 22540225.

\section{References}

[1] V. Guillemin and S. Sternberg, Geometric Asymptotics, Math. Surveys, No.14, Amer. Math. Soc., Providence, 1977.

[2] J. E. Marsden, T. S. Ratiu, Introduction to mechanics and symmetry, Springer-Verlag, New York, 1994.

[3] K. Efstathiou and D.A. Sadovdskii, Normalization and global analysis of perturbations of the hydrogen atom by weak electric and magnetic fields, Rev. Mod. Phys., 82, 2099-2154 (2010).

[4] D. A. Sadovskií, B. I. Ẑhilinskií, Tuning the hydrogen atom in crossed fields between the Zeeman and Stark limits, Phys. Rev. A 57, 2867-2884 (1998).

[5] R. H. Cushman, D. A. Sadovskií, Monodromy in perturbed Kepler systems: Hydrogen atom in crossed fields, Europhys. Lett., 47 (1), 1-7 (1999).

[6] R. H. Cushman, D. A. Sadovskií, Monodromy in the hydrogen atom in crossed fields, Physica D 142, 166-196 (2000). 
[7] C. R. Schleif and J. B. Delos, Monodromy and the structure of the energy spectrum of hydrogen in near perpendicular electric and magnetic fields, Phys. Rev. A 76, 013404 (2007).

[8] B. K. Efstathiou, D. A. Sadovskií, and B. I. Zhilinskii, Classification of perturbations of the hydrogen atom by small static electric and magnetic fields, Proc. R. Soc. A 463, 1771-1790 (2007).

[9] C. R. Schleif and J. B. Delos, Semiclassical theory of the structure of the hydrogen spectrum in near-perpendicular electric and magnetic fields: Derivations and formulas for Einstein-Brillouin-Keller-Maslov quantization and description of monodromy, Phys. Rev. A 77, 043422 (2008).

[10] K. Efstathiou, O. V. Lukina, and D. A. Sadovskií, Most typical 1:2 resonant perturbation of the hydrogen atom by weak electric and magnetic fields. Phys. Rev. Lett. 101, 253003 (2008).

[11] K. Efstathiou, O. V. Lukina, and D. A. Sadovskií, Complete classification of qualitatively different perturbations of the hydrogen atom in weak nearorthogonal electric and magnetic fields, J. Phys. A: math. Theor. 42, 055209 (2009).

[12] T. Iwai, A dynamical group $S U(2,2)$ and its use in the MIC-Kepler problem, J. Phys. A: Math. Gen. 26, 609-630 (1993).

[13] H. V. McIntosh and A. Cisneros, Degeneracy in the presence of a magnetic monopole, J. Math. Phys. 11, 896-916 (1970).

[14] T. Iwai and N. Katayama, Two classes of dynamical systems all of whose bounded trajectories are closed, J. Math. Phys. 35, 2914-2933 (1994).

[15] T. Iwai, A gauge theory for the quantum planar three-body problem, J. Math. Phys. 28, 964-974 (1987).

[16] G. D. Birkoff, Dynamical Systems, A.M.S. Colloquium Publications, vol.IX, New York, 1927.

[17] F. G. Gustavson, On constructing formal integrals of a Hamiltonian system near an equilibrium, Astron. J. 71, 670-686 (1966).

[18] J. K. Moser, Lectures on Hamiltonian systems, Memoirs of A. M. S. 81, Providence, 1968. 
[19] M. Robnik, The algebraic quantization of the Birkhoff-Gustavson normal form, J. Phys. A: Math. Gen. 17, 109-130 (1984).

[20] T. Iwai, Y. Uwano, The four-dimensional conformal Kepler problem reduces to the three-dimensional Kepler problem with a centrifugal potential and Dirac's monopole field. Classical theory, J. Math. Phys. 27, 1523-1529 (1986).

[21] R. Cushman, D. L. Rod, Reduction of the semisimple 1:1 resonance, Physica D 6, 105-112 (1982).

[22] T. Iwai, On reduction of two degrees of freedom Hamiltonian systems by an $S^{1}$ action, and $S O_{0}(1,2)$ as a dynamical group, J. Math. Phys. 26 885-893 (1985).

[23] Y. Uwano, From the Birkhoff-Gustavson normalization to the BertrandDarboux integrability condition, J. Phys. A: Math. Gen. 33 6635-6653 (2000).

[24] R. Simon, S. Chaturvedi, V. Srinivasan, and N. Mukunda, Hamilton's turns for the Lorentz group, Internat. J. Theret. Phys., 45, 2075-2094 (2006).

[25] J. Rawnsley and S. Sternberg, On representations associated to the minimal nilpotent coadjoint orbit of $S L(3, \mathbb{R})$, Amer. J. Math., 104, 1153-1180 (1982).

[26] A. Smirnov, Two-body systems from $S L(2, \mathbb{C})$-tops, aiXiv:0711.2432v1 [math.DS] 15 Nov 2007. 